\title{
Molecular gas and a new young stellar cluster in the far outer Galaxy ${ }^{\star \star \star}$
}

\author{
J. L. Yun, D. Elia, P. M. Palmeirim, J. I. Gomes, and A. M. Martins
}

\begin{abstract}
Universidade de Lisboa - Faculdade de Ciências, Centro de Astronomia e Astrofísica da Universidade de Lisboa, Observatório Astronómico de Lisboa, Tapada da Ajuda, 1349-018 Lisboa, Portugal

e-mail: [yun; eliad;ppalm; jgomes; amartins]@oal.ul.pt
\end{abstract}

Received 20 November 2008 / Accepted 26 March 2009

ABSTRACT

\begin{abstract}
Aims. We investigate the star-formation ocurring in the region towards IRAS 07527-3446 in the molecular cloud [MAB97]250.633.63 , in the far outer Galaxy. We report the discovery of a new young stellar cluster, and describe its properties and those of its parent molecular cloud.

Methods. Near-infrared $J H K_{\mathrm{S}}$ images were obtained with VLT/ISAAC, and millimetre line CO spectra were obtained with the SEST telescope. VLA archive date were also used.

Results. The cloud and cluster are located at a distance of $10.3 \mathrm{kpc}$ and a Galactocentric distance of $15.4 \mathrm{kpc}$, in the far outer Galaxy. Morphologically, IRAS 07527-3446 appears as a young embedded cluster of a few hundred stars seen towards the position of the IRAS source, extending for about 2-4 pc and exhibiting sub-clustering. The cluster contains low and intermediate-mass young reddened stars, a large fraction having cleared the inner regions of their circumstellar discs responsible for $\left(H-K_{\mathrm{S}}\right)$ colour excess. The observations are compatible with a $\leq 5 \mathrm{Myr}$ cluster with variable spatial extinction of between $A_{\mathrm{V}}=5$ and $A_{\mathrm{V}}=11$. Decomposition of $\mathrm{CO}$ emission in clumps, reveals a clump clearly associated with the cluster position, of mass $3.3 \times 10^{3} M_{\odot}$. Estimates of the slopes of the $K_{\mathrm{S}}$-band luminosity function and of the star-formation efficiency yield values similar to those seen in nearby star-formation sites. These findings reinforce previous results that the distant outer Galaxy continues to be active in the production of new and rich stellar clusters, with the physical conditions required for the formation of rich clusters continuing to be met in the very distant environment of the outer Galactic disc.
\end{abstract}

Key words. stars: formation - ISM: clouds - ISM: individual objects: IRAS 07527-3446 - infrared: stars - ISM: dust, extinction stars: pre-main sequence

\section{Introduction}

Stars form in molecular clouds across the Galaxy. Most molecular gas and dust exist in the Galactic disc and are most abundant toward the inner Galaxy (Clemens et al. 1988). Thus, most starformation sites, containing mostly young stellar clusters, have been found and studied in regions of the inner disk and in nearby molecular clouds (e.g., Tapia et al. 1991; Strom et al. 1993; McCaughrean \& Stauffer 1994; Horner et al. 1997; Luhman et al. 1998).

Young stellar clusters are gravitationally bound systems of stars that formed from the same parent cloud. They usually represent a statistically significant sample of stars with similar distances, and chemical compositions. Quite frequently, they exhibit a relatively wide range of masses, often containing main-sequence massive stars as well as low-mass pre-mainsequence stars. A main piece of information to understand the star-formation process is the stellar mass distribution that results from the fragmentation of molecular clouds. Whether it is universal or depends on local environments, initial mass, or luminosity functions of a young cluster can be used to address important questions about the star-formation process (e.g., Pandey et al. 2005). Young embedded clusters, in particular,

\footnotetext{
^ Based on observations collected at the ESO 8.2-m VLT-UT1 Antu telescope (program 66.C-0015A).

$\star \star$ Table 2 is only available in electonic form at

http://www . aanda.org
}

unlike more evolved open clusters, which have lost significant numbers of low-mass stars due to a combination of cluster dynamics and Galactic tides (Vesperini \& Heggie 1997), can be used to probe the stellar initial mass function ina more accurate (e.g., Muench et al. 2000), and its possible variation in space and time.

Because stellar mass is not a directly observable quantity, the luminosity function has been most extensively used to infer the underlying mass function (Luhman et al. 2000; Muench et al. 2003). For embedded clusters, the near-infrared luminosity function is most adequate as it can be obtained directly from photometric observations of the stellar cluster members, whose spectral energy distributions peak in the near or mid-infrared, without the assumption of any theoretical mass-luminosity-age relations and their corresponding uncertainties.

Some attention has been devoted to star-formation sites toward the outer Galaxy and at large Galactocentric distances (defined as $>13.5 \mathrm{kpc}$; this number is based on the radial distribution of CO emission from the studies of Digel et al. 1996; Heyer et al. 1998). Extensive studies were performed of the distribution of $\mathrm{CO}$, and of $\mathrm{H}_{2}$, in star-formation sites in the outer Galaxy as well as a comparison of molecular clouds across the Galaxy (Wouterloot \& Brand 1989; Wouterloot et al. 1990; Brand \& Wouterloot 1995). Based on observations in the millimetre and radio frequencies (basically molecular gas lines, maser emission, and ionized gas continuum emission), several star-formation sites in the far outer Galaxy were found (e.g. 
Fich \& Blitz 1984; Wouterloot et al. 1988; Rudolph et al. 1996). As for the stellar content of star-formation sites in the far outer Galaxy, discovery of isolated young stellar objects located at an estimated Galactocentric distance of about 15-19 kpc were reported by Kobayashi \& Tokunaga (2000). Santos et al. (2000) reported the discovery of two of the most distant galactic young stellar clusters (located at a distance of $10.2 \mathrm{kpc}$ and a Galactocentric distance of $16.5 \mathrm{kpc}$ ) embedded in a molecular cloud containing a CS dense core. Similarly, Snell et al. (2002) identified 11 stellar clusters in the second Galactic quadrant with Galactocentric distances of between 13.5 and $17.3 \mathrm{kpc}$. Yun et al. (2007) reported the discovery of an embedded cluster of an age of about 4 Myr at a Galactocentric distance of $15 \mathrm{kpc}$ in the outer Galaxy. Brand \& Wouterloot (2007) found an embedded cluster of about 60 stars at a Galactocentric distance of $20 \mathrm{kpc}$. Yasui \& Kobayashi (2008) studied the stellar content of two embedded clusters near the edge of the Galaxy.

However, there are still relatively few known resolved young embedded stellar clusters in the far outer Galaxy. This has prevented a systematic analysis of the star-formation properties in regions very far from the Galactic center. These regions have lower metallicities, lower gas densities and lower ambient pressures, which could affect the star-formation process resulting in different rates of cloud evolution. A different distribution of stellar masses in a cluster (different IMF) could emerge and consequently a different feedback mechanism from the stellar to the molecular component.

As part of our study of very distant young embedded clusters in the third Galactic quadrant, we report here the discovery of a new young stellar cluster embedded in a molecular cloud [MAB97]250.63-3.63 (May et al. 1997), located far in the outer Galaxy, seen towards IRAS 07527-3446. At a distance of about $10.3 \mathrm{kpc}$, it is one of the most distant young embedded clusters known to date.

Section 2 describes the observations and data reduction. In Sect. 3, we present and discuss the results. A summary is given in Sect. 4.

\section{Observations and data reduction}

\subsection{Near-infrared observations}

Near-infrared $\left(J, H\right.$ and $\left.K_{\mathrm{S}}\right)$ images were obtained on 2000 November 11 using the ESO Antu (VLT Unit 1) telescope equipped with the short-wavelength arm (Hawaii Rockwell) of the ISAAC instrument. The ISAAC camera (Moorwood et al. 1998 ) contains a $1024 \times 1024$ pixel near-infrared array and was used at a plate scale of $0.147 \mathrm{arcsec} /$ pixel resulting in a field of view of $2.5 \times 2.5 \mathrm{arcmin}^{2}$ on the sky. For each filter, 6 dithered sky positions were observed. Series of 15 images with individual on-source integration time of $4 \mathrm{~s}$ were taken in the $J$-band. Similarly, series of 20 images, each of 3-s integration time, were obtained in the $H$ and $K_{\mathrm{S}}$ bands.

The images were reduced using a set of our own IRAF scripts to correct for bad pixels, subtract the sky foreground, and flatfield the images. Dome flats were used to correct for the pixel-topixel variations in the response. The selected images were then aligned, shifted, trimmed, and co-added to produce a final mosaic image for each band $J H K_{\mathrm{S}}$. Correction for bad pixels was made while constructing the final mosaics, which cover about $3 \times 3 \operatorname{arcmin}^{2}$ on the sky. Due to the presence of significant field distortion in these ISAAC images (two pixels at the edges and 2.5 pixels in the corners), the individual tiles of mosaics were distortion corrected before they were tiled together. The correction for field distortion was performed using IRAF/geotran together with the adequate correction files provided by ESO at their web page.

The central, coadded region, with enhanced signal-to-noise ratio covers about $2.8 \times 2.8 \operatorname{arcmin}^{2}$ on the sky. Point sources were extracted using daofind with a detection threshold of $4 \sigma$. The images were inspected to look for false detections that had been included by daofind in the list of detected sources. These sources were eliminated from the source list and a few additional sources were added in by hand. Aperture photometry was made with a small aperture (radius $=2$ pix, which is about the measured $F W H M$ of the point spread function) and aperture corrections, found from bright and isolated stars in each image, were used to correct for the flux lost in the wings of the PSF. The error in the determination of the aperture correction was $<0.05$ mag in all cases. A total of 630, 548, and 732 sources were found to have fluxes in $J, H$, and $K_{\mathrm{S}}$, respectively, and errors $\sigma_{K \mathrm{~s}}<0.15 \mathrm{mag}$ in the $K_{\mathrm{S}}$ band.

The $J H K_{\mathrm{S}}$ zero points were determined using faint infrared standard stars (S677-D and P545-C of the LCO/Palomar NICMOS Table of Photometric Standards; see Persson et al. 1998) and checked with those obtained using 2MASS stars brighter than $K_{\mathrm{S}}=13.5 \mathrm{mag}$ from the 2MASS All-Sky Release Point Source Catalogue (Skrutskie et al. 2006; Cutri et al. 2003). Photometry errors are in the range from about 0.08 mag for the bright stars to $0.15 \mathrm{mag}$ for the fainter ones. From the histograms of the $J H K_{\mathrm{S}}$ magnitudes, we estimate the completeness limit of the observations to be roughly $20.0 \mathrm{mag}$ in the $J$-band, and 18.5 magnitudes in the $H$, and $K_{\mathrm{S}}$ bands.

\section{2. $\mathrm{CO} \mathrm{mm}$ line observations}

Millimetre single-dish observations of IRAS 07527-3446 were carried out at the $15 \mathrm{~m}$ SEST telescope (ESO, La Silla, Chile), during two observational campaigns, in 2001 May and 2002 December, respectively. Three maps were obtained in the rotational lines of ${ }^{12} \mathrm{CO}(1-0)(115.271 \mathrm{GHz}),{ }^{13} \mathrm{CO}(1-0)$ (110.201 GHz), and ${ }^{13} \mathrm{CO}(2-1)(220.399 \mathrm{GHz})$, respectively. The SEST half-power beam width (HPBW) was $46^{\prime \prime}$ at $110 \mathrm{GHz}$ and $22^{\prime \prime}$ at $230 \mathrm{GHz}$, so that, since the adopted grid spacing was $46^{\prime \prime}$, the ${ }^{13} \mathrm{CO}(2-1)$ line emission was strongly undersampled. The ${ }^{12} \mathrm{CO}(1-0)$ and ${ }^{13} \mathrm{CO}(2-1)$ maps are composed of $10 \times 8$ pointings, while the ${ }^{13} \mathrm{CO}(1-0)$ map is smaller $(6 \times 7$ pointings). These sizes correspond, on the sky, to an area markedly larger than the field of the VLT images, as discussed below. Attempts were made to detect the emission from the $\mathrm{C}^{18} \mathrm{O}(1-0)$, $\mathrm{C}^{18} \mathrm{O}(2-1), \mathrm{CS}(2-1), \mathrm{CS}(3-2)$ transitions toward the central (IRAS source) position, using $t_{\text {int }}=180 \mathrm{~s}$, but no spectral line was found.

The adopted integration time was $t_{\text {int }}=60 \mathrm{~s}$ for each pointing. A high-resolution 2000 channel acousto-optical spectrometer was used as a back end, with a total bandwidth of $86 \mathrm{MHz}$ and a channel width of $43 \mathrm{kHz}$; it was split into two halves to measure both the 115 and $230 \mathrm{GHz}$ receivers simultaneously (in particular, the ${ }^{12} \mathrm{CO}(1-0)$ and the ${ }^{13} \mathrm{CO}(2-1)$ lines were observed simultaneously, as were the ${ }^{13} \mathrm{CO}(1-0)$ and $(2-1)$ lines). At these frequencies, the aforementioned channel width corresponds to approximately $0.11 \mathrm{~km} \mathrm{~s}^{-1}$ and $0.055 \mathrm{~km} \mathrm{~s}^{-1}$, respectively. The spectra were taken in frequency-switching mode, recommended to save observational time when mapping extended sources. The antenna temperature was calibrated with the standard chopper wheel method. Pointing was checked regularly towards known circumstellar $\mathrm{SiO}$ masers; pointing accuracy was estimated to be higher than $5^{\prime \prime}$. 
The data-reduction pipeline consisted of the following steps: i) folding the frequency-switched spectrum; ii) fitting the baseline by a high-order polynomial and subtracting it; iii) coadding repeated spectra obtained at the same sky position, weighting by both the integration time and the inverse of the system temperature; iv) obtaining the main beam temperature $T_{\mathrm{MB}}$ by dividing the antenna temperature $T_{\mathrm{A}}$ by the $\eta_{\mathrm{MB}}$ factor, equal to 0.7 for the first two lines (at $110-115 \mathrm{GHz}$ ) and 0.5 for the third one (at $220 \mathrm{GHz}$ ); and v) finally, rebinning the spectra to obtain the same LSR velocity grid for all the lines, with an exact channel separation $\Delta V_{\text {chann }}=0.12$ and $0.06 \mathrm{~km} \mathrm{~s}^{-1}$ at 115 and $230 \mathrm{GHz}$, respectively.

The spectrum baseline RMS noise (in $T_{\mathrm{MB}}$ ), averaged over all map positions, has been found to be $0.2 \mathrm{~K}$ for ${ }^{12} \mathrm{CO}(1-0)$ and ${ }^{13} \mathrm{CO}(1-0)$, and $0.15 \mathrm{~K}$ for ${ }^{13} \mathrm{CO}(2-1)$.

The same data-reduction pipeline was applied to the spectra of the aforementioned transitions with no detection, in order to evaluate the values of the rms noise to be adopted as a $1 \sigma$ upper limit for those lines. The values obtained are $0.1 \mathrm{~K}$ for $\mathrm{C}^{18} \mathrm{O}(1-0), 0.2 \mathrm{~K}$ for $\mathrm{C}^{18} \mathrm{O}(2-1), 0.09 \mathrm{~K}$ for $\mathrm{CS}(2-1)$, and $0.07 \mathrm{~K}$ for $\mathrm{CS}(3-2)$.

\subsection{VLA centimetre observations}

We searched for continuum observations made with the Very Large Array (VLA) of the National Radio Astronomy Observatory (NRAO) ${ }^{1}$ to look for a radio continuum counterpart to IRAS 07527-3446. The VLA data archive contained continuum observations at $6 \mathrm{~cm}$ towards IRAS 07527-3446, which were carried out with the VLA in its C and D configurations on 1993 October 3. The observations were made in both left and right circular polarization, with an effective bandwidth of $100 \mathrm{MHz}$. The phase centre of the observations was $\alpha(2000)=$ $7^{\mathrm{h}} 54^{\mathrm{m}} 37.2^{\mathrm{s}}, \delta(2000)=-34^{\circ} 54^{\prime} 49^{\prime \prime} .0$. The data were reduced and calibrated using the Astronomical Image Processing System (AIPS) software of the NRAO. The flux calibrator was 3C 286, with an adopted flux density of $7.5 \mathrm{Jy}$, while the phase calibrator was 0826-373, with a bootstrapped flux density of $2.3 \pm 0.01 \mathrm{Jy}$. The image had an rms sensitivity of $160 \mu \mathrm{Jy} /$ beam, with a size $(F W H M)$ and position angle (PA) of the synthesized beam of $18^{\prime \prime} .0 \times 13^{\prime \prime} .1$ and $-23^{\circ}$, respectively.

\section{Results and discussion}

\subsection{The young stellar cluster: identification and morphology}

Figure 1 presents the ISAAC $J H K_{\mathrm{S}}$ colour composite image obtained towards IRAS 07527-3446. A reddened stellar cluster is clearly seen in the northern part of the image, where the presence of a high concentration of stars resides. In this final, mosaicked image, IRAS 07527-3446 (and the cluster it represents) is not located at the central position. This is the result of a deliberate choice for the position of the centre of the initial frames, about $1^{\prime}$ south of the IRAS source nominal position. This choice was made to avoid contamination of the images by an extremely bright, unrelated star (HD 64876: $K_{\mathrm{S}}=2.6, \mathrm{~V}=6.1$ ) located about $4^{\prime}$ northward of the IRAS source.

A simple visual inspection of Fig. 1 reveals that the stars appear to form two distinct groups or sub-clusters: a northeastern (NE) group and a southwestern (SW) group. The SW group appears to be richer with a more significant central concentration

1 The National Radio Astronomy Observatory is a facility of the National Science Foundation operated under cooperative agreement by Associated Universities, Inc.

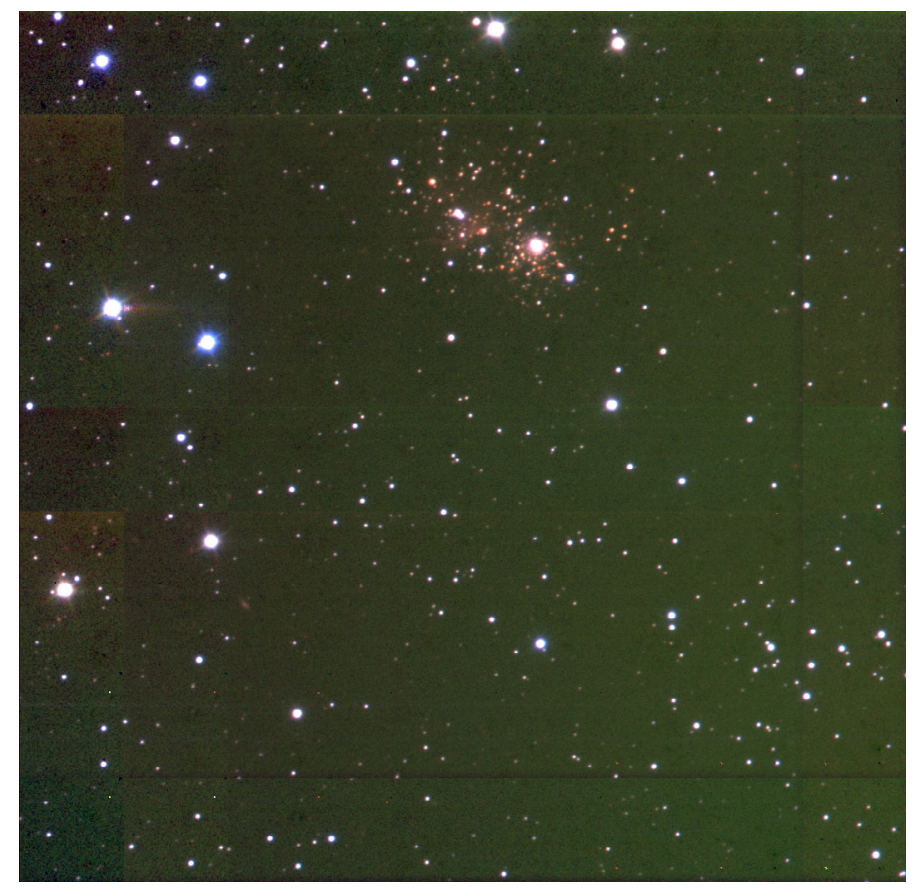

Fig. 1. $J$ (blue), $H$ (green), and $K_{\mathrm{S}}$ (red) colour composite image towards IRAS 07527-3446. A new young red cluster is seen in the northern part of the image. The image covers about $2.8 \times 2.8 \mathrm{arcmin}^{2}$. North is up and East to the left.

of stars where the brightest star is located. The NE group appears less extended and more sparse but also redder. In addition, the NE group includes the presence of tenuous diffuse extended emission. This can be seen more clearly in Fig. 2. The lower panel of this figure shows what we define below as the "cluster region". A careful analysis of this panel reveals the presence of faint diffuse extended (nebular) emission, seen mostly towards the NE group.

In order to detect the presence of the cluster and any possible sub-clustering in a more robust way, a contour plot of the stellar surface density was made and is shown in Fig. 3. The contour levels are set to show significant enhancements above the average field star density. In this figure, a clear concentration in the spatial distribution of stars is seen, which is identified as a stellar cluster. The contours marking this cluster are elongated in a direction close to SW-NE (position angle of about $55^{\circ}$ ). The concentration of stars at the strongest peak of the surface stellar density of the image (corresponding to the SW group) is very high ( 900 stars $\mathrm{pc}^{-2}$; for a distance of $10.3 \mathrm{kpc}$, see below), about 60 times higher than the average field star density (which itself was determined by counting stars in the southern part of the image where the cluster is not located). Away from this peak, in the transversal direction (perpendicular to the elongation), the cluster merges with the field for distances of the order of $16^{\prime \prime}$ ( $\sim 0.8 \mathrm{pc}$, see below). The cluster appears to occupy an area, which when approximated by an ellipse, has major and minor axes of 2.3 and $1.7 \mathrm{pc}$, respectively. Down to the $K_{\mathrm{S}}$-band magnitude limit of 18.5, the mean surface density in this cluster (after subtraction of the average field star density of $14 \mathrm{pc}^{-2}$ ) is 45 stars $\mathrm{pc}^{-2}$. Within the ellipse defined above, the expected number of cluster members is $\sim 138$ stars, and the expected number of field stars is $\sim 44$ stars.

A second peak is also clearly present in the surface stellar density map. It corresponds to the NE group of stars and its peak value is two-thirds of the stronger SW peak. 


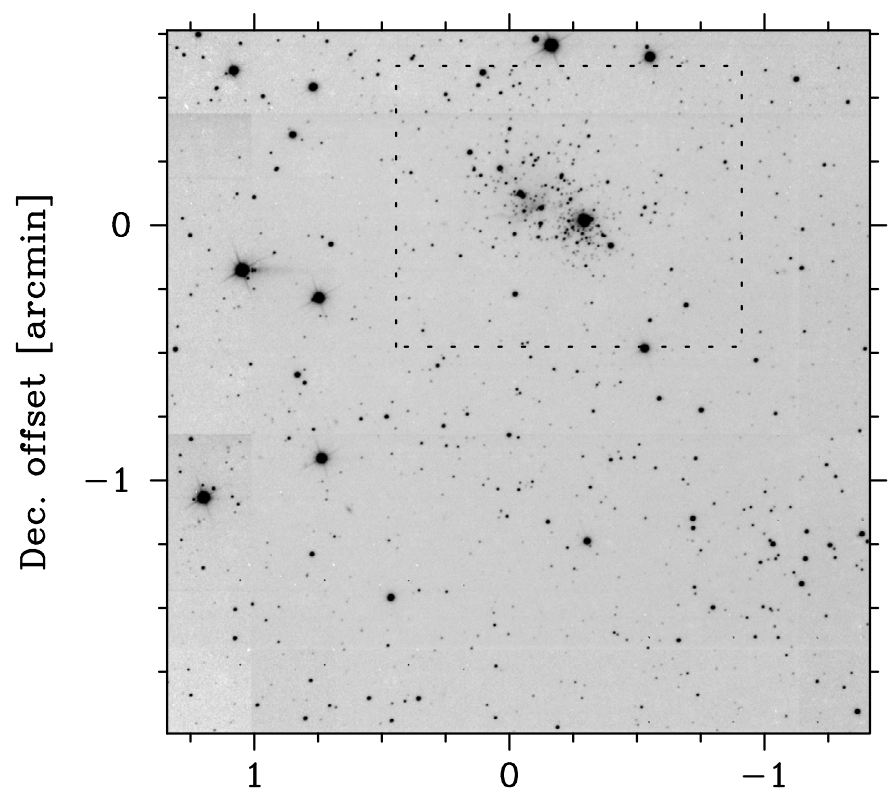

R.A. offset [arcmin]

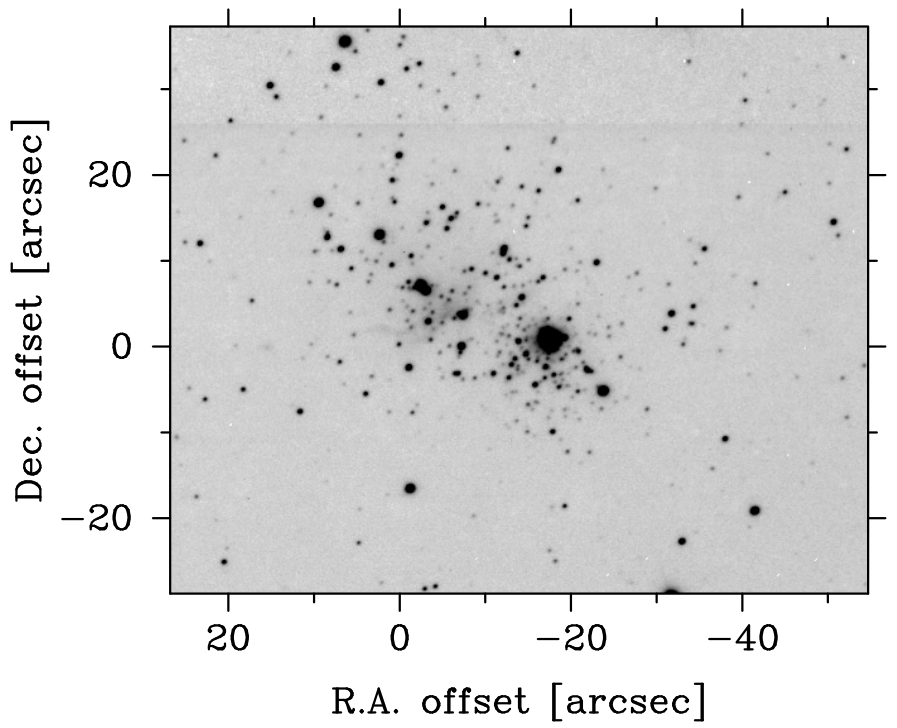

Fig. 2. $K_{\mathrm{S}}$-band image towards IRAS 07527-3342. The dashed box indicates the "cluster region (see Sect. 3.3.1)" seen in more detail in the lower panel (close-up view of $81^{\prime \prime} \times 66^{\prime \prime}$ containing the cluster). The axes give the coordinates relative to the IRAS source $(\alpha(2000)=$ $\left.7^{\mathrm{h}} 54^{\mathrm{m}} 37.2^{\mathrm{s}}, \delta(2000)=-34^{\circ} 54^{\prime} 49^{\prime \prime} .0\right)$. North is up and East to the left.

\subsection{The molecular cloud}

\subsubsection{Molecular gas and distance}

The IRAS 07527-3446 position falls within the centroid of a CO cloud listed in May et al. (1997), of their catalog of southern outer Galaxy molecular clouds (for this cloud, hereafter we adopt the SIMBAD nomenclature [MAB97]250.63-3.63). Our $\mathrm{CO}$ maps presented here represent a portion of this cloud, observed with unprecedented resolution, considering that the sampling interval of May et al. (1997) was 7'.5. The CO maps are significantly larger than the field imaged in the NIR, giving information about the gas distribution in that zone on a large scale, the cluster itself being covered only by the $(0,0)$ and $(1,0)$ pointings.

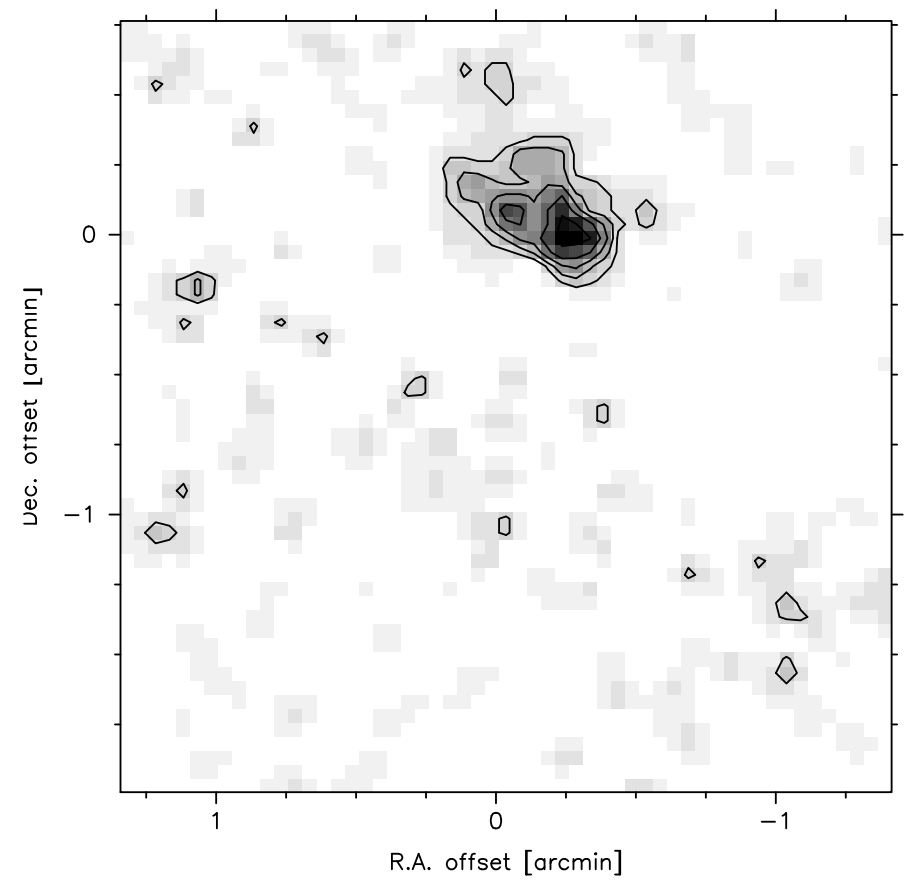

Fig. 3. Contour plot of the stellar surface density towards IRAS $07257-$ 3446. The contour levels are $3,6,10,15$, and $22 \sigma$ above the background mean stellar density. A main stellar cluster is easily identified with evidence for sub-clustering. Offsets are relative to the IRAS source $\left(\sigma=40 \mathrm{stars} \mathrm{pc}^{-2}\right.$, for a distance of $\left.10.3 \mathrm{kpc}\right)$.
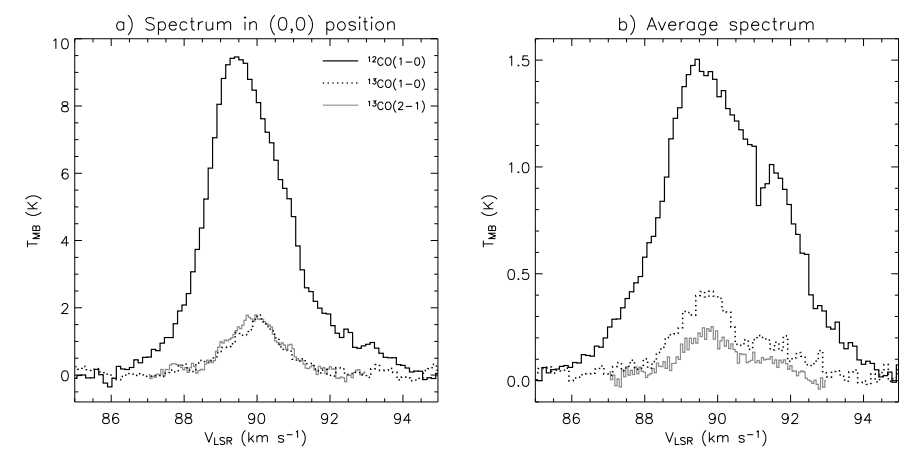

Fig. 4. Panel a): spectra of the three observed lines towards the IRAS source $((0,0)$ position of the maps). Panel $\mathbf{b})$ : average of all spectra in the map, for each line.

Only lines characterized by a signal-to-noise ratio $S N R>3$ were considered as genuine emission. Out of the 80 observed points, significant ${ }^{12} \mathrm{CO}(1-0)$ and ${ }^{13} \mathrm{CO}(2-1)$ emission was detected from 43 and 26 of them, respectively, while for ${ }^{13} \mathrm{CO}(1-0)$ emission was detected from 18 pointings out of 42 .

Figure 4 (panel a) shows the spectra taken towards IRAS 07527-3446 $\left((0,0)\right.$ position of the map). The ${ }^{12} \mathrm{CO}(1-0)$ spectrum peaks at about $89 \mathrm{~km} \mathrm{~s}^{-1}$, while those of both ${ }^{13} \mathrm{CO}$ lines peak at about $90 \mathrm{~km} \mathrm{~s}^{-1}$; these values can be adopted as the velocity of the gas component associated with the cluster, and are consistent with the global radial velocity of [MAB97]250.63-3.63 quoted by May et al. (1997). The bulk of the $\mathrm{CO}$ emission is in the range $V_{\mathrm{lsr}} \simeq 85-95 \mathrm{~km} \mathrm{~s}^{-1}$ for the ${ }^{12} \mathrm{CO}(1-0)$ line, and $V_{\mathrm{lsr}} \simeq 88-93 \mathrm{~km} \mathrm{~s}^{-1}$ for the other two lines (Fig. 4, panel b).

The first piece of information that can be extracted from the spectra is the kinematic heliocentric distance, derived from the 


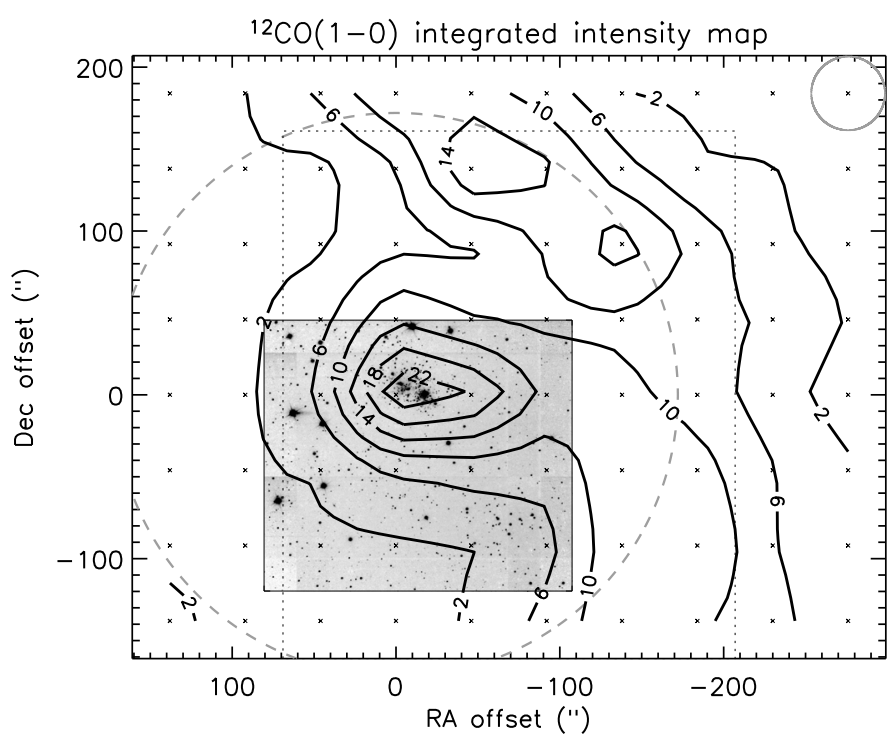

Fig. 5. Smoothed contours of ${ }^{12} \mathrm{CO}(1-0)$ integrated intensity (in the velocity range between 84 and $96 \mathrm{~km} \mathrm{~s}^{-1}$ ) observed in the IRAS 07527 3446 region, superimposed on the $K$-band image of Fig. 2 . The lowest contour is at $2 \mathrm{~K} \mathrm{~km} \mathrm{~s}^{-1}(8 \sigma)$. Subsequent contours are in steps of $4 \mathrm{~K}$ $\mathrm{km} \mathrm{s}^{-1}$. The SEST beam at $115 \mathrm{GHz}$ is displayed in the top right corner. The dotted box delimits the area observed in ${ }^{13} \mathrm{CO}(1-0)$. The grey dashed circle represents approximatively the position and the average size of the gas clump (centred on the cluster position) obtained by decomposing the cloud with CLUMPFIND (see text). The small crosses indicate the observed positions.

peak radial velocity. This can be done applying the circular rotation model by Brand \& Blitz (1993). Using $V_{\mathrm{LSR}}=90 \mathrm{~km} \mathrm{~s}^{-1}$, a distance $d \simeq 10.3 \mathrm{kpc}$ is obtained. Given the Galactic longitude, this implies a Galactocentric distance of $15.4 \mathrm{kpc}$ to the cloud, well into the far outer Galaxy and among the largest for known Galactic star-formation sites. We estimate a distance uncertainty of up to $20 \%$ due to uncertainties in the rotation curve and possible streaming motions.

\subsubsection{Molecular gas distribution}

The contour map of the ${ }^{12} \mathrm{CO}(1-0)$ integrated intensity $I=$ $\int T_{\mathrm{MB}} \mathrm{d} v$ is shown in Fig. 5, superimposed on the $K$-band image. The ${ }^{13} \mathrm{CO}(1-0)$ and ${ }^{13} \mathrm{CO}(2-1)$ integrated intensity contour maps are shown in Fig. 6.

The morphology of the emission is very similar in all the maps, with the main (strongest) peak located at the cluster location. The cluster appears to correspond to a strong gas concentration which emerges from a more diffuse filament. In more peripheral zones of the maps, it is possible to recognize the presence of more condensations, one north of the cluster, and another southwest of it, as highlighted by Figs. 5 and 6, and discussed in detail below.

Channel (velocity) maps were built from the line observations (Figs. 7 and 8), where these "clumps" are seen to correspond to different velocity components. The clump in the northern part of the map is responsible for the second peak (at $V_{\mathrm{LSR}}=91.5 \mathrm{~km} \mathrm{~s}^{-1}$ ) in the average spectrum in Fig. 4, panel b). In terms of radial velocity, the central condensation corresponding to the cluster exhibits an intermediate velocity position.

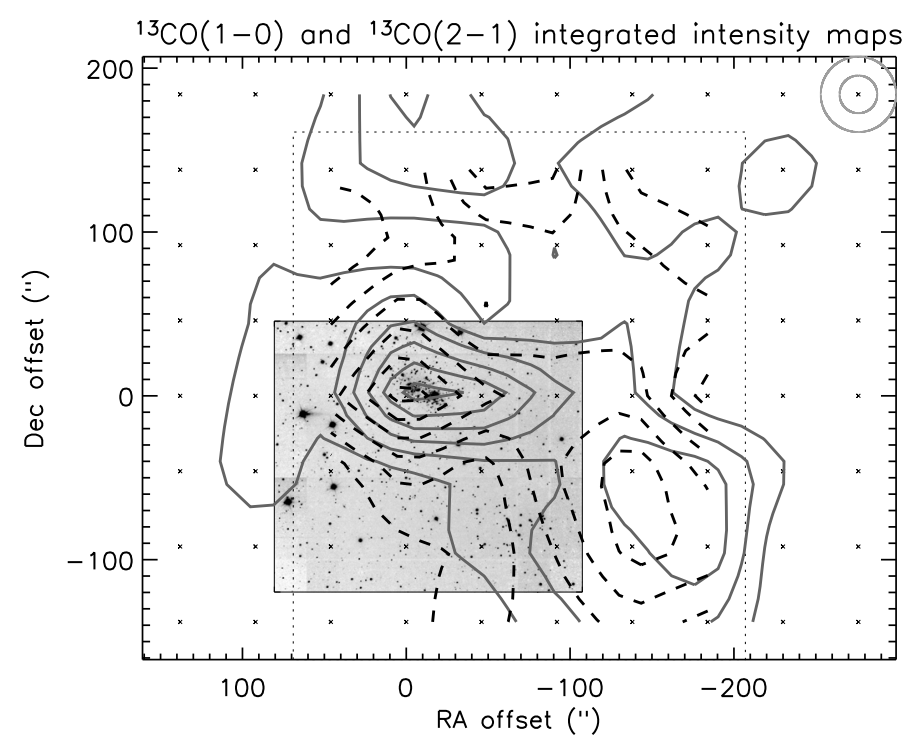

Fig. 6. As in Fig. 5, but for ${ }^{13} \mathrm{CO}(1-0)$ (dashed line) and ${ }^{13} \mathrm{CO}(2-1)$ (grey solid line) transitions. In both cases, levels start at $0.5 \mathrm{~K} \mathrm{~km} \mathrm{~s}^{-1}$ (2.5 $\sigma$ for ${ }^{13} \mathrm{CO}(1-0)$ and $5.5 \sigma$ for $\left.{ }^{13} \mathrm{CO}(2-1)\right)$ and are in steps of $0.5 \mathrm{~K} \mathrm{~km} \mathrm{~s}^{-1}$; they are not labeled for the sake of clarity. The range of velocities are between 85 and $95 \mathrm{~km} \mathrm{~s}^{-1}$ for ${ }^{13} \mathrm{CO}(1-0)$ and between 87 and $93 \mathrm{~km} \mathrm{~s}^{-1}$ for ${ }^{13} \mathrm{CO}(2-1)$. The SEST beams at 110 and $220 \mathrm{GHz}$ are displayed in the top right corner by means of a large and a small grey circle, respectively. The dotted box delimits the area observed in ${ }^{13} \mathrm{CO}(1-0)$.

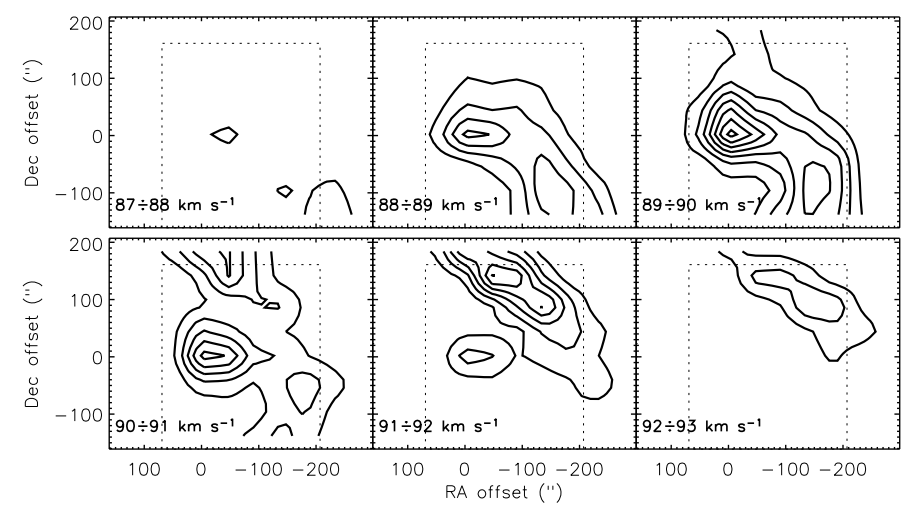

Fig. 7. Channel maps obtained integrating the ${ }^{12} \mathrm{CO}(1-0)$ emission over velocity ranges of width $1 \mathrm{~km} \mathrm{~s}^{-1}$. Contours are in steps of $1 \mathrm{~K} \mathrm{~km} \mathrm{~s}^{-1}$. Dashed line delimits the area observed also in ${ }^{13} \mathrm{CO}(1-0)$.
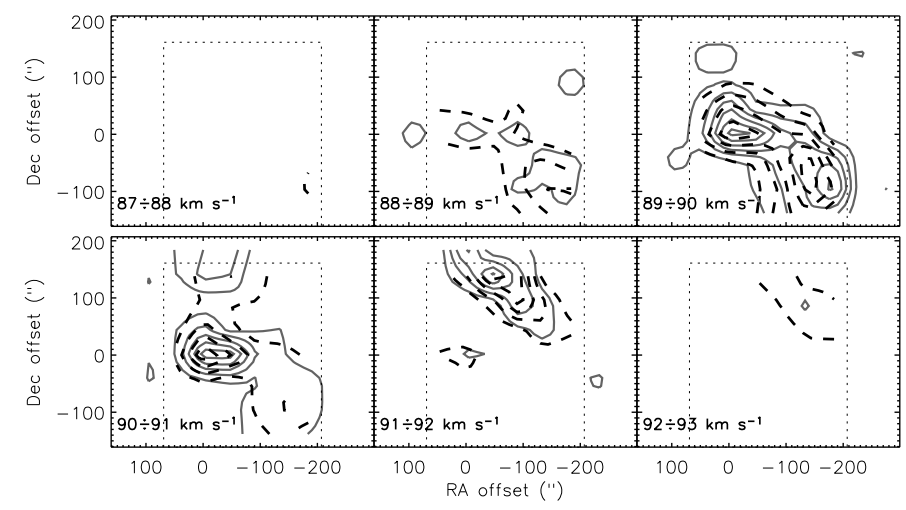

Fig. 8. As in Fig. 7, but for ${ }^{13} \mathrm{CO}(1-0)$ (dashed line) and ${ }^{13} \mathrm{CO}(2-1)$ (grey solid line). Contours are in steps of $0.2 \mathrm{~K} \mathrm{~km} \mathrm{~s}^{-1}$. 


\subsubsection{Molecular cloud mass and optical extinction}

A few different methods can be exploited to obtain an estimate of the cloud mass from the line emission of molecular tracers, in this case the $\mathrm{CO}$ isotopes. A first estimate can be made using the empirical correlation between the ${ }^{12} \mathrm{CO}(1-0)$ integrated intensity and the $\mathrm{H}_{2}$ column density along the line of sight, $N_{i, j}$, at position $(i, j)$ :

$N_{i, j}\left(\mathrm{H}_{2}\right)=X \int T_{\mathrm{MB}} \mathrm{d} v \quad\left(\mathrm{~cm}^{-2}\right)$,

where the constant $X$ has been determined empirically. Several authors quote different values for $X$, and it is accepted that, in general, it should vary with the Galactic position. In the southern outer Galaxy, including the [MAB97]250.63-3.63 cloud, May et al. (1997) used a value of $X=3.8 \times 10^{20} \mathrm{~cm}^{-2}\left(\mathrm{~K} \mathrm{~km} \mathrm{~s}^{-1}\right)^{-1}$, which is almost twice the values typically adopted for nearer clouds. Indeed, Strong \& Mattox (1996) derived a revised value for $X$, of $1.9 \times 10^{20} \mathrm{~cm}^{-2}\left(\mathrm{~K} \mathrm{~km} \mathrm{~s}^{-1}\right)^{-1}$; it should be corrected according to Brand \& Wouterloot (1995), who argued that in the far outer Galaxy it may be $30-45 \%$ higher than the local/inner Galaxy value. Thus, in this case we adopt $X=$ $2.8 \times 10^{20} \mathrm{~cm}^{-2}\left(\mathrm{~K} \mathrm{~km} \mathrm{~s}^{-1}\right)^{-1}$. To obtain the mass from the column density, we also used a value of the mean molecular mass $\mu$ corrected for the gradient in He abundance along the Galactic radial direction; at a Galactocentric distance of $R=17 \mathrm{kpc}$, a correction of 1.28 on the mass value has to be applied to take in account this variation, and consequently $\mu$ varies from his "typical" value of 2.72 (Allen 1973) to 2.56 there (Wilson \& Matteucci 1992). Using these corrected values, we obtain a mass of $M_{\mathrm{CO}}=1.3 \times 10^{4} M_{\odot}$ for the mapped area.

This estimate is in good agreement with the upper limit represented by the value of $3.2 \times 10^{4} M_{\odot}$ obtained by May et al. (1997) for the [MAB97]250.63-3.63 total mass using the same method. Scaled to their values of X and $\mu$, we find $1.7 \times 10^{4} M_{\odot}$.

Furthermore, the derived $N_{0,0}\left(\mathrm{H}_{2}\right)$ value, of the column density towards the map reference position, can be used to calculate the optical extinction $A_{\mathrm{V}}$ (see Bohlin et al. 1978). Using the relation $N\left(\mathrm{H}_{2}\right) / A_{\mathrm{V}}=9.4 \times 10^{20} \mathrm{~cm}^{-2} \mathrm{mag}^{-1}$ (Frerking et al. 1982), we obtain $A_{\mathrm{V}}=7.8 \mathrm{mag}$, compatible with the value derived from infrared colour excesses (Sect. 3.2.2).

Another method for estimating the cloud mass consists of assuming LTE conditions and using both an optically thick line and an optically thin line, in this case ${ }^{12} \mathrm{CO}(1-0)$, and ${ }^{13} \mathrm{CO}(1-0)$ or ${ }^{13} \mathrm{CO}(2-1)$, respectively. In this method (see e.g., Pineda et al. 2008, for details), the peak main beam temperature of the ${ }^{12} \mathrm{CO}(1-0)$ line is used to derive its excitation temperature, which, for example, is found to be $12.5 \mathrm{~K}$ at the map reference position. Then, assuming that excitation temperatures are the same for both lines, the optical depth and the column density of ${ }^{13} \mathrm{CO}$ are calculated for each line of sight (see also Elia et al. 2007, for the specific case of ${ }^{13} \mathrm{CO}(2-1)$ ). Finally, to calculate the total mass, it is necessary to adopt $\mathrm{a} \mathrm{H}_{2} /{ }^{13} \mathrm{CO}$ abundance ratio, which in the far outer is expected to be greater than the "typical" value of $5 \times 10^{5}$ quoted by Dickman (1978); Brand \& Wouterloot (1995) indeed found that for $R=17 \mathrm{kpc}$, a ratio of $3 \times 10^{6}$ should be used.

The ${ }^{13} \mathrm{CO}(2-1)$ line corresponds more faithfully to the optically thin case, and moreover the map extent coincides with that of the ${ }^{12} \mathrm{CO}(1-0)$ map, so that a direct comparison is possible. The mismatch between the SEST beam size at this frequency and the adopted map spacing was fixed by assigning the integrated intensity at each position to the entire grid element, effectively replacing the smaller ${ }^{13} \mathrm{CO}(2-1)$ beam size by the ${ }^{12} \mathrm{CO}(1-0)$ beam size. The obtained mass amounts to $M_{13} \mathrm{CO}(2-1)=7.6 \times 10^{3} M_{\odot}$, which is compatible with that obtained by means of the previous empirical method. The remaining discrepancy can be explained by taking into account that the number of usable ${ }^{13} \mathrm{CO}(2-1)$ spectra, contributing to the total map mass estimate, is markedly lower than in the case of ${ }^{12} \mathrm{CO}(1-0)$, due to lower $S / N$, and taking into account that the LTE approximation method seems to typically underestimate the ${ }^{13} \mathrm{CO}$ true column densities (Padoan et al. 2000). Finally, we should note that these two lines, ${ }^{12} \mathrm{CO}(1-0)$ and ${ }^{13} \mathrm{CO}(2-1)$, map different parts of the cloud because of their different optical depths, and also that, evidently, the empirical method based on the ${ }^{12} \mathrm{CO}(1-0)$ emission is quite reliable in a statistical way, but for individual clouds it may give inaccurate results. Therefore, given these considerations, the two mass estimates derived here can be considered to be in good agreement.

The column density of ${ }^{13} \mathrm{CO}$, derived from ${ }^{13} \mathrm{CO}(2-1)$, at the centre position can also be used to estimate the optical extinction $A_{\mathrm{V}}$ towards the cluster by applying a linear relation:

$A_{\mathrm{V}}=c_{1} N\left({ }^{13} \mathrm{CO}\right)+c_{2}$.

Several estimates of the $c_{1}$ and $c_{2}$ parameters can be found in the literature, varying with the observed region and the calibration technique. Here, we adopt the results of Frerking et al. (1982) for the Taurus region, $c_{1}=7.1 \times 10^{-16} \mathrm{mag} \mathrm{cm}^{-2}$ and $c_{2}=1.0$ mag respectively. Thus, the obtained visual extinction estimate is $A_{\mathrm{V}}=2.3 \mathrm{mag}$, much smaller than the value obtained from the ${ }^{12} \mathrm{CO}(1-0)$ data.

Repeating the same procedure, now for the ${ }^{13} \mathrm{CO}(1-0)$ line, but with the aforementioned caveats about optical thickness and map size, and about the accuracy of the LTE technique, we obtain a total mass $M_{{ }^{13} \mathrm{CO}(1-0)}=8.6 \times 10^{3} M_{\odot}$, and a visual extinction of $A_{\mathrm{V}}=3.1 \mathrm{mag}$ toward the cluster line of sight.

\subsubsection{Structure of the gas emission}

Once derived the global characteristics of the region mapped in $\mathrm{CO}$, we focus on the structure of the gas emission, analysing in particular the cloud component that appears to be spatially associated with the IRAS 07527-3446 cluster. This can be done by applying a cloud decomposition algorithm that analyses the RADec- $V_{\text {LSR }}$ data cube, assigning channels of observed spectra to different "clumps", and estimates the masses of these clumps separately. A typical choice is the CLUMPFIND code (Williams et al. 1994); even though some problems can arise when applying this algorithm to very complex velocity fields (strong dependence from the initial setup, see e.g., discussion in Elia et al. 2007), in this case the input data cube is such that the cloud decomposition turned out to be stable. Because of the higher $S / N$ in the spectra, the ${ }^{12} \mathrm{CO}(1-0)$ data cube was chosen for decomposition. The input data requested by the algorithm are the radiation temperature threshold to start the search for clumps, and the level increment. These were defined to be: $T_{\min }=0.5 \mathrm{~K}$, and $\Delta T=0.5 \mathrm{~K}$, in both cases more than two times higher than the rms noise of the line, as suggested by Williams et al. (1994).

Four clumps were found, and in particular one of them (hereafter clump A) is centred on the strongest peak of the map, i.e. the cluster position. The output average radius provided by CLUMPFIND is drawn in Fig. 5, as a dashed circle that encloses the entire area imaged in NIR. The properties of all four clumps are summarized in Table 1 . In positions where they spatially overlap, spectral channels are attributed to different velocity components and then assigned to different clumps. Because 
Table 1. Properties of ${ }^{12} \mathrm{CO}(1-0)$ detected clumps.

\begin{tabular}{lcccccc}
\hline \hline Clump & $\begin{array}{c}\text { Map offset } \\
\left({ }^{\prime \prime},{ }^{\prime \prime}\right)\end{array}$ & $\begin{array}{c}\text { Diameter } \\
(\mathrm{pc})\end{array}$ & $\begin{array}{c}V_{\mathrm{LSR}} \\
\left(\mathrm{km} \mathrm{s}^{-1}\right)\end{array}$ & $\begin{array}{c}\Delta V \\
\left(\mathrm{~km} \mathrm{~s}^{-1}\right)\end{array}$ & $\begin{array}{c}M_{12 \mathrm{CO}} \\
\left(M_{\odot}\right)\end{array}$ & $\begin{array}{c}M_{\text {vir }} \\
\left(M_{\odot}\right)\end{array}$ \\
\hline $\mathrm{A}$ & $(0,0)$ & 18.7 & 89.4 & 1.24 & $3.3 \times 10^{3}$ & $2.7 \times 10^{3}$ \\
$\mathrm{~B}$ & $(-46,+132)$ & 19.1 & 91.4 & 1.68 & $4.0 \times 10^{3}$ & $5.2 \times 10^{3}$ \\
$\mathrm{C}$ & $(-132,-92)$ & 16.2 & 89.2 & 0.99 & $2.1 \times 10^{3}$ & $1.5 \times 10^{3}$ \\
$\mathrm{D}$ & $(-230,-132)$ & 15.7 & 87.8 & 0.81 & $0.8 \times 10^{3}$ & $1.0 \times 10^{2}$ \\
\hline
\end{tabular}

of the small extent of the map, all the clumps are flagged by the algorithm as containing pixels that lie on the edge of the investigated area and are expected to extend outside it as well, and their average size is evaluated in this perspective. Clumps B, C, and, mainly, D are more affected by this uncertainty. Applying to clump A the empirical method as above, we a mass estimated $M_{A}$ to be $3.3 \times 10^{3} M_{\odot}$. This value can be considered as the mass of the gas component presently associated with the cluster, as obtained directly from ${ }^{12} \mathrm{CO}(1-0)$ brightness.

Using the average velocity dispersion provided by the output of CLUMPFIND, and combining this with the estimated average radius, the virial mass for these clumps is estimated and given by:

$M_{\mathrm{vir}}=210\left(\frac{R}{\mathrm{pc}}\right)\left(\frac{\Delta V}{\mathrm{~km} \mathrm{~s}^{-1}}\right)^{2} \quad\left(M_{\odot}\right)$,

where the multiplicative constant corresponds to the case of a uniform clump density (for density profiles varying as $\rho \propto r^{-1}$ or as $\rho \propto r^{-2}$, the constant is replaced by 190 and 126, respectively). Virial masses of the four identified clumps are listed in Table 1. For all these clumps, the ratio of virial masses to those obtained from the ${ }^{12} \mathrm{CO}(1-0)$ integrated intensity is within 20 $30 \%$ of unity. This situation is then compatible with the virial equilibrium; however, the uncertainties in these mass estimates are too large to allow a robust conclusion.

Finally, the excitation temperature $T_{\mathrm{ex}}$ can be derived from the line intensity ratio of a molecular isotope, under the assumption of optically thin and LTE regime. For the two ${ }^{13} \mathrm{CO}$ lines at the $(0,0)$ position of the map, the line ratio is $\sim 1$ (see also Fig. 4, panel $a$ ) and we derive an excitation temperature $T_{\text {ex }}=7.7 \mathrm{~K}$. On the other hand, as written above, in the LTE assumption it is also possible to derive the excitation temperature from the thick line of ${ }^{12} \mathrm{CO}(1-0)$, and in this point we obtain $T_{\mathrm{ex}}=12.5 \mathrm{~K}$. Both these values are consistent with the range of 6-15 K found by Brand \& Wouterloot (1995) for far outer Galaxy clouds, for which these authors do not note significant differences with those present in the inner Galaxy part. On the other hand, Mead \& Kutner (1988) proposed a different scenario, with a temperature gradient and lower temperatures in the outer Galaxy, being able to reproduce their data with a value of $7 \mathrm{~K}$, not significantly different from that we obtain from the ratio of the ${ }^{13} \mathrm{CO}$ lines.

\subsection{The stellar content}

\subsubsection{Reddening and cluster membership}

The top panel of Fig. 9 shows the spatial distribution of the $\left(H-K_{\mathrm{S}}\right)$ colour across the image, as a function of the RA offset relative to the lower left (SE corner) of Fig. 2. The bottom panel is the same but as a function of Dec. offset. The redder values of $\left(H-K_{\mathrm{S}}\right)$ seen for values of RA offset between $54^{\prime \prime}$ and $135^{\prime \prime}$ and Dec. offset between $91^{\prime \prime}$ and $157^{\prime \prime}$ indicate that a large number

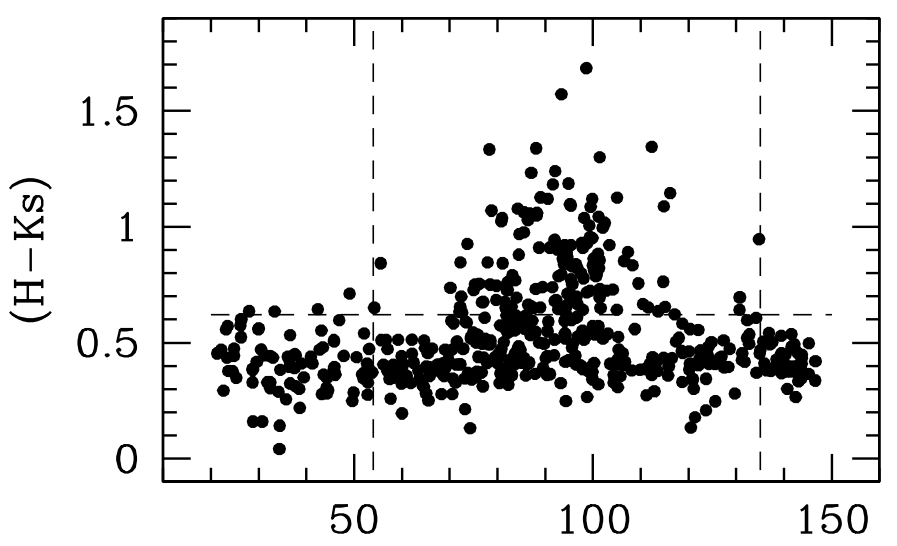

R. A. Offset (")

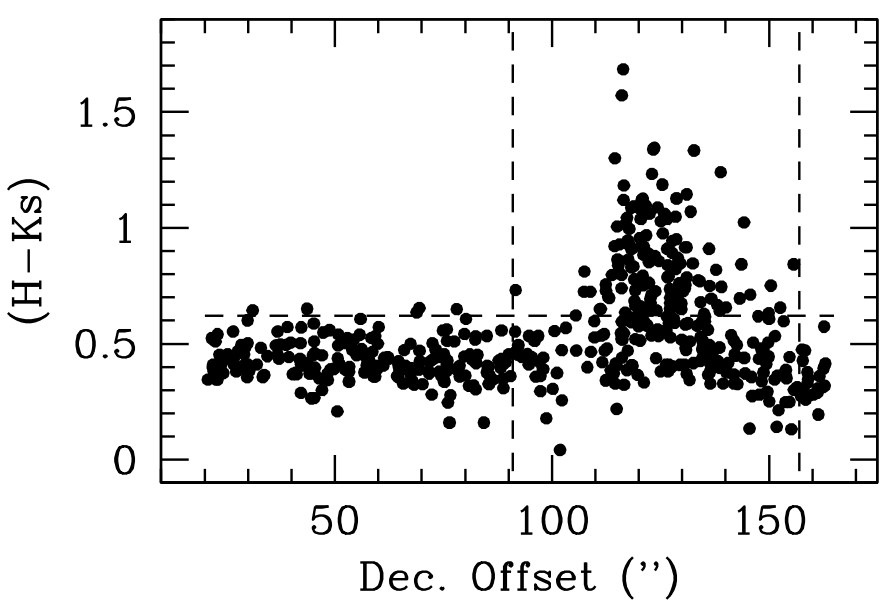

Fig. 9. a) (Top): Plot of the observed $\left(H-K_{\mathrm{S}}\right)$ colour as a function of the RA offset relative to the lower left (SE corner) of the upper panel of Fig. 2. The reddening effect $\left(\left(H-K_{\mathrm{S}} \geq 0.62\right.\right.$, horizontal dashed line indicating the field star average plus $2 \sigma$ ) of a dense cloud core is clearly seen for values of RA offsets between approximately $54^{\prime \prime}$ and $135^{\prime \prime}$ (indicated by vertical dashed lines). b) (Bottom): The same as $a$ but as a function of Dec offset. The presence of the dense cloud core occurs for Dec offsets between approximately $91^{\prime \prime}$ and 157".

of sources in this region exhibit colour excesses with respect to field stars. These ranges of coordinates define a box that we refer to as the "cluster region" (shown as the dashed box in Fig. 2). In the cluster region, there is an enhancement in the visual extinction traced by redder $(H-K \mathrm{~s})$ colours, corresponding to the presence of the cluster parental molecular cloud. This signals the embedded nature and youth of the cluster sources.

We plot in Fig. 10 the histograms of the $\left(H-K_{\mathrm{S}}\right)$ colours of the sources detected in both the $H$ and $K_{\mathrm{S}}$-band images. Sources closer than about $20^{\prime \prime}$ to the left or the lower borders were excluded. The solid line represents the histogram of 258 sources located inside the cluster region. The mean value of their $\left(H-K_{\mathrm{S}}\right)$ 


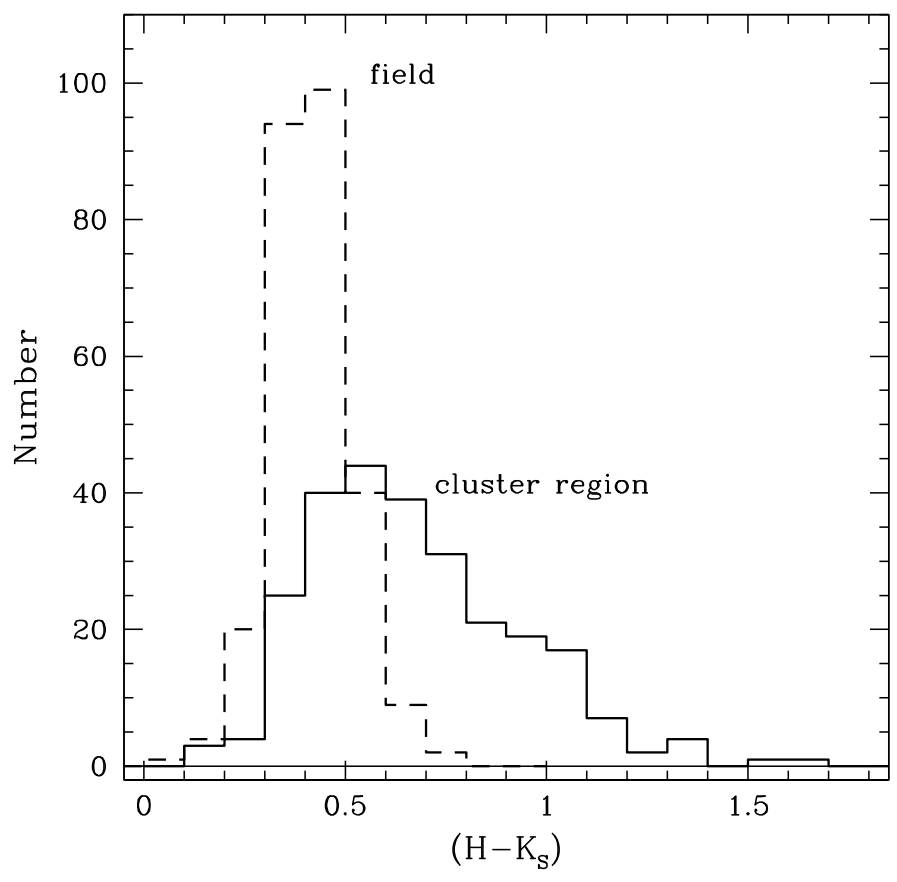

Fig. 10. Histogram of the observed $\left(H-K_{\mathrm{S}}\right)$ colours for sources inside (solid line) or outside (dashed line) the cluster region.

colours is 0.67 and the standard deviation is 0.26 . The dashed line corresponds to the histogram of the 269 sources located outside the cluster region (contained mostly in the southern part of the image). Here, the sources, mostly foreground field sources, exhibit a close to symmetrical histogram and have very different statistical values: their mean value is $\left(H-K_{\mathrm{S}}\right)_{\text {field }}=0.42$, with a standard deviation of 0.10 . These values were used to draw the horizontal line in Fig. 9, which is placed at a value of $\left(H-K_{\mathrm{S}}\right)=0.62$, i.e., at a value of the mean of $\left(H-K_{\mathrm{S}}\right)_{\text {field }}$ plus $2 \sigma$.

As argued in the next paragraphs, we consider a sufficient condition for cluster membership that a star is located inside the cluster region and has a colour of $(H-K) \geq 0.62$.

Along the horizontal axis of Fig. 9, for all values of RA, there are values of $\left(H-K_{\mathrm{S}}\right)$ between about 0.05 and 0.62 . The reddening effect of the cloud clearly stands out as enhanced values of $\left(H-K_{\mathrm{S}}\right)$ for values of RA offset between approximately $54^{\prime \prime}$ and $135^{\prime \prime}$ (a range of $81^{\prime \prime}$ - about $4 \mathrm{pc}$ at the source distance of $10.3 \mathrm{kpc}$ - indicated by the two vertical dashed lines). A similar conclusion is reached by analysis of the values of $\left(H-K_{\mathrm{S}}\right)$ as a function of Dec offset. For the general case, this colour excess of $\left(H-K_{\mathrm{S}}\right)=0.62$ would just separate the objects into two groups: a group of foreground stars and a group of \{embedded + background stars. However, given that at this distance and location in the Galaxy, there are very few background stars, this reddening effect separates foreground from embedded stars and thus effectively selects cluster members.

Consequently, we take the diagrams of Fig. 9 and the association of $\mathrm{CO}$ emission as evidence of a cloud core and we use it to define the "cluster region" as this rectangular region of about $81^{\prime \prime} \times 66^{\prime \prime}$ on each side. The cluster region is shown in the close-up panel of Fig. 2. A star that is located in the cluster region and has $\left(H-K_{\mathrm{S}}\right)>0.62$ is considered here to be a cluster member. Evidently, this is a sufficient but not a necessary condition. There may be additional cluster members outside the cluster region or inside it with bluer values of $\left(H-K_{\mathrm{S}}\right)$. One

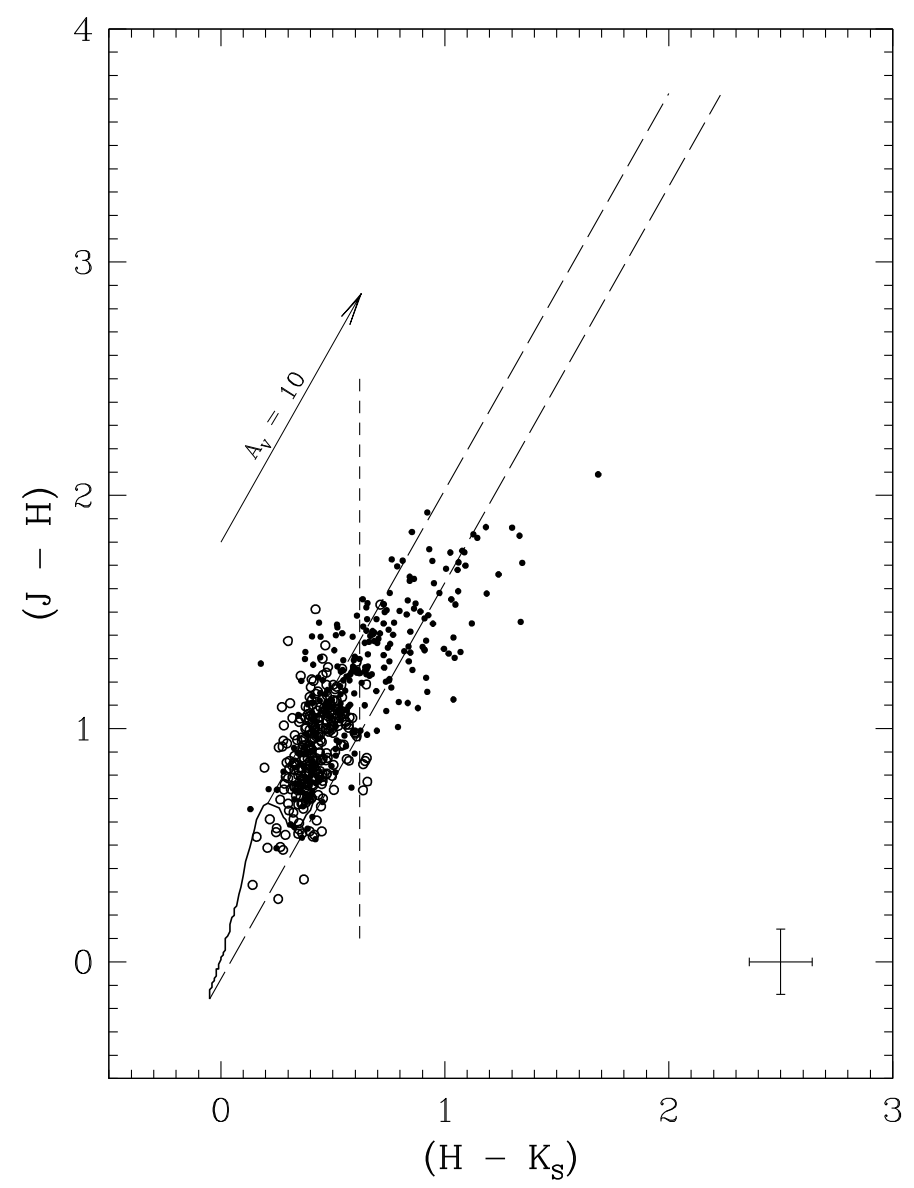

Fig. 11. Near-infrared colour-colour diagram of the region towards IRAS 07527-3446 seen in Fig. 2. Filled circles represent the sources contained in the "cluster region" (see text). Open circles are sources outside the cluster region. The solid line represents the loci of unreddened main-sequence stars (Bessel \& Brett 1988) while long-dash lines indicate the reddening band. The reddening vector indicates the direction of the shift produced by extinction by dust with standard properties (Rieke \& Lebofsky 1985). The location of the vertical dashed line, derived from Fig. 9, is at $\left(H-K_{\mathrm{S}}\right)=0.62$. Notice that virtually all the sources to the right of this line are in the cluster region. The cross in the lower right corner represents a typical error bar.

hundred and thirty-six sources verify these criteria and are thus good candidate cluster members. This number is in good agreement with the expected number of 138 cluster members derived from the surface density plot in Sect. 3.1. It represents a lower limit to the number of young stellar cluster members detected in the $H$ and $K_{\mathrm{S}}$-band images. Photometry of these sources is given in Table $2^{2}$. In this table, Cols. (1)-(3) identify the stars giving, respectively, the running ID number, and the equatorial coordinates (RA and Dec epoch 2000); Cols. (4)-(6) give the photometric indices $K_{\mathrm{S}},\left(H-K_{\mathrm{S}}\right)$, and $\left(J-K_{\mathrm{S}}\right)$.

\subsubsection{Young stellar objects}

In the region shown in the top panel of Fig. 2, using the 500 point sources detected in all three $J, H$, and $K_{\mathrm{S}}$-bands, we have plotted the near-infrared colour-colour diagram, $(J-H)$ versus $\left(H-K_{\mathrm{S}}\right)$. This is shown in Fig. 11. In this plot, filled circles represent sources contained in the $81^{\prime \prime} \times 66^{\prime \prime}$ central region

2 Table 2 is available in electronic form at EDP. 
("cluster region", where most sources are likely to be members of the cluster). Open circles are sources outside this region. The location of the vertical dashed line, derived from Fig. 9, is at $\left(H-K_{\mathrm{S}}\right)=0.62$. Most of the sources to the right of this line $\left(\left(H-K_{\mathrm{S}}\right)>0.62\right)$ are in the cluster region. Sources to the left of the dotted line are both inside and outside the cluster region.

The solid line represents the loci of unreddened mainsequence stars (Bessel \& Brett 1988). Most stars are located within the reddening band (long-dash lines), where stars appear if they are main-sequence stars reddened according to the interstellar extinction law (Rieke \& Lebofsky 1985), which defines the reddening vector (traced here for $A_{\mathrm{V}}=10$ ). Pre-mainsequence YSOs that have had time to clear the inner regions of their circumstellar discs lie in this region as well. Giant stars appear slightly above this band. Stars located to the right of the reddening band are likely to be embedded young star objects with infrared excess emission due to the presence of circumstellar material (Adams et al. 1987).

We note that not many sources lie in the infrared excess region. Among the sources with values $\left(H-K_{\mathrm{S}}\right) \geq 0.62$, the fraction of sources in the infrared excess region is $40 \%$. This relatively low number of sources in this region suggests that most sources $(60 \%)$ have dissipated the inner parts of their circumstellar discs that are responsible for the $\left(H-K_{\mathrm{S}}\right)$ excess. This result indicates that the age of this cluster is likely to be about 3-5 Myr. This is because inner disc frequencies in young clusters have been found to decrease steadily as the cluster ages approach about 6 Myr (Haisch et al. 2001), with about 50\% of the sources exhibiting excess emission from circumstellar discs at $3 \mathrm{Myr}$ and very few sources at ages older than about $6 \mathrm{Myr}$. The cluster could be younger if it contains Class II sources with circumstellar discs that cannot be detected in a JHK colour-colour diagram (Lada \& Lada 1995).

For the sources that lie inside the reddening band, the highest value of $\left(H-K_{\mathrm{S}}\right)$ is about 1.08 . Using the mean value of $\left(H-K_{\mathrm{S}}\right)=0.42$ for field stars (according to Fig. 10), we obtain a colour excess $E\left(H-K_{\mathrm{S}}\right)=0.66$ due to intra-cloud extinction, which corresponds to about a maximum visual extinction produced by the cloud core of about $A_{\mathrm{V}}=11$ (Rieke \& Lebofsky 1985).

An additional estimate of the age (or range of ages) for this cluster can be obtained from the colour-magnitude diagram, $K_{\mathrm{S}}$ vs. $\left(H-K_{\mathrm{S}}\right)$, plotted in Fig. 12, where open circles have the same meaning as in Fig. 11, and filled circles are as in Fig. 11 but exclude sources with circumstellar emission (outside the reddening band). The two dashed lines are the zero-age main-sequence (ZAMS) for an arbitrary distance with zero extinction and with $A_{\mathrm{V}}=2.8$, respectively. This extincted ZAMS represents a rough fit to the open circles (which are spread mostly along a nearly vertical band). The several brightest sources, which are clearly blue, are field giants (non-cluster members). The large spread in the open circles around the fit is expected since the foreground stars are located at variable distances and their different lines-ofsight may be affected by variable values of ISM extinction. Thus, this value of extinction $\left(A_{\mathrm{V}}=2.8\right)$ represents a rough lower limit to the extinction in the region: it is caused by the foreground diffuse ISM, and does not include the internal cloud extinction.

Focusing our attention now on the assumed cluster members (filled circles), the location of a source on this colourmagnitude diagram is the result of a combination of cluster distance, cluster age, and extinction along the line-of-sight towards the source. Assuming a fixed distance for the cluster members, vertical spread (scatter in magnitude $K_{\mathrm{S}}$ ) can be caused mostly by a spread in age. Variable extinction will only produce a small

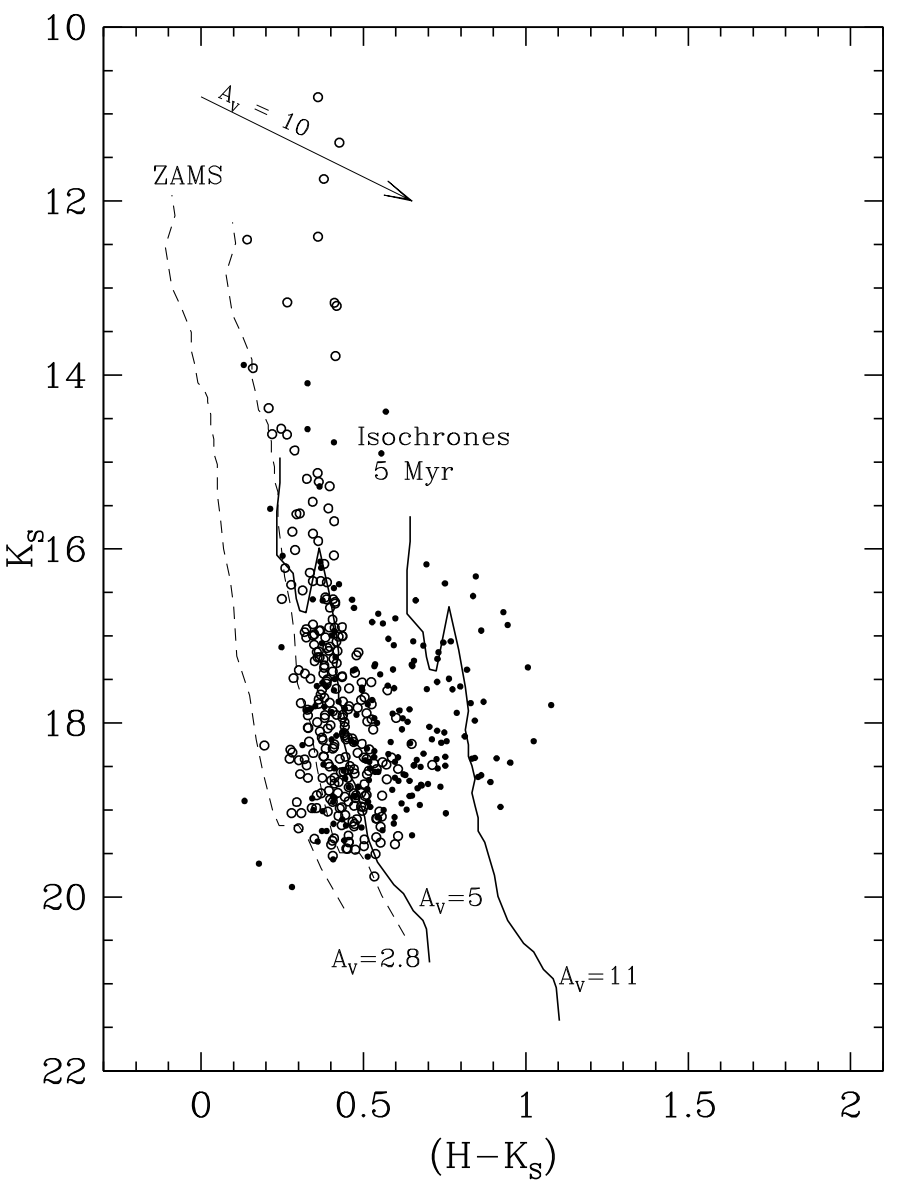

Fig. 12. Colour-magnitude diagram of the region towards IRAS 075273446 seen in Fig. 2. Filled circles and open circles have the same meaning as in Fig. 11 but excluding sources with circumstellar emission. The two dashed lines are, respectively, the zero-age main-sequence (ZAMS, Siess et al. 2000) for an arbitrary distance with zero extinction and with $A_{\mathrm{V}}=2.8$. The two solid lines are the $5 \mathrm{Myr}$ isochrone with extinctions $A_{\mathrm{V}}=5$ and $A_{\mathrm{V}}=11$, respectively. The reddening vector indicates the direction of the shift produced by extinction by dust with standard properties (Rieke \& Lebofsky 1985).

vertical dispersion because the extinction vector is almost horizontal (see Fig. 12). On the other hand, a horizontal spread in this diagram (scatter in colour) can be due to different ages, variable spatial extinction, or a combination of both. The existence of variable extinction, with highly different amounts of dust across young clusters is expected. Non-coeval star-formation cannot be excluded either. Thus, we have tried to fit the filled circles using pre-main-sequence isochrones reddened by variable amounts of extinction.

Fitting pre-main-sequence isochrones to young stellar clusters is a rather uncertain process but it can give a rough estimate of the age or a range of ages of a young cluster. We have used the kinematic distance of $10.3 \mathrm{kpc}$ and the set of Siess et al. (2000) isochrones with masses ranging from 0.1 to $7 M_{\odot}$. Given the absence of metallicity information, we have adopted solar metallicity. A set of isochrones of different ages and extinctions were generated and plotted in the colour-magnitude diagram. Figure 12 shows a possible fit to the filled circles: an isochrone of $5 \mathrm{Myr}$ with extinctions varying from $A_{\mathrm{V}}=5$ to $A_{\mathrm{V}}=11$, in good agreement with the values of $A_{\mathrm{V}}$ derived from the ${ }^{12} \mathrm{CO}$ gas column densities. The uncertainty in the isochrone models imply an age spread of about 2 Myr. The value of $A_{\mathrm{V}} \sim 5$ for 
the least-extincted cluster sources is in good agreement with the value of $\left(H-K_{\mathrm{S}}\right)=0.62$ for the same sources. Using an intrinsic (non-reddened) average $\left(H-K_{\mathrm{S}}\right)$ colour of about 0.25 for field stars (mostly main-sequence and red giants; Koornneef 1983; Bessel \& Brett 1988), the colour excess $E\left(H-K_{\mathrm{S}}\right)=$ $0.62-0.25=0.37$ corresponds to an $A_{\mathrm{V}}=15.3 \times E\left(H-K_{\mathrm{S}}\right)=5.6$ (Rieke \& Lebofsky 1985). Isochrones of younger ages will fit only the lower mass-end (the fainter sources). They would require the absence of any intermediate-mass stars restricting the cluster population to low-mass stars only. Assuming an age of $5 \mathrm{Myr}$ for this cluster, and considering the faintest cluster member, the lowest mass detected in our $K_{\mathrm{S}}$-band image is about $0.4 M_{\odot}$ for $A_{\mathrm{V}}=5$, and about $0.8 M_{\odot}$ for $A_{\mathrm{V}}=11$. Similarly, the highest-mass star present in our image is estimated to be between 5 and $9 M_{\odot}$. This value is in good agreement with the absence of massive stars inferred from the lack of VLA continuum emission.

According to the VLA $6 \mathrm{~cm}$ observations (Sect. 2.3), and down to the sensitivity of these observations, no radiocontinuum emission was detected at this wavelength. Setting the lower-limit detection for the $6 \mathrm{~cm}$ continuum emission to be at $4 \sigma$, and adopting a distance of $10.3 \mathrm{kpc}$, the rate of ionizing photons required to maintain the ionization of a possible HII region up to that level is $\dot{N}_{i} \simeq 6.7 \times 10^{45} \mathrm{~s}^{-1}$ (under the assumption of an optically thin, homogeneous spherical HII region with constant temperature of $T=10^{4} \mathrm{~K}$; e.g., Rodríguez et al. 1980). This implies an upper limit of $\sim 6 \times 10^{3} L_{\odot}$ (Panagia 1973, corresponding to a B1 zero-age main-sequence star with $M \sim 12 M_{\odot}$ ) for the luminosity of the individual members of the IRAS 07527-3446 cluster.

Furthermore, the luminosity of the cluster region is dominated by the mid and far-infrared flux as measured by IRAS. We estimate this $L_{\mathrm{FIR}}$ to be about $3.5 \times 10^{3} L_{\odot}$. Even assuming (unrealistically) that all this luminosity is produced by a single star, this would set an upper limit of about $8 M_{\odot}$ for any massive star present in this cluster, again in good agreement with the above estimates.

\subsubsection{Luminosity function}

The $K_{\mathrm{S}}$-band luminosity function of this source was constructed using the results of our $K_{\mathrm{S}}$-band photometry. The cluster members were grouped into bins of 0.4 mag width. Figure 13 shows the results. The upper left panel gives the histogram of $K_{\mathrm{S}}$ magnitude for all sources in the image, whereas the upper right panel is the histogram for all sources located inside the cluster region. In an attempt to correct for foreground field stars, we present in the lower left panel the histogram of the reddest sources inside the cluster region, which should be composed of essentially cluster members. No attempt has been made to deredden the sources. A different attempt to correct for the foreground stars consisted of considering the southern part of the image (where few or no cluster members exist) as a reference field. The histogram of the reference field (not shown) was scaled to the area of the cluster region and subtracted from the histogram of the cluster region yielding the lower right panel.

We consider the straight line $\left(\log N=a+b K_{\mathrm{S}}\right)$ that best fits each histogram in the range of bins, where there are statistically significant numbers of sources $\left(K_{\mathrm{S}} \sim 16-19\right)$. Interestingly, the slopes $a$ of the four histograms, in that range of bins, are very similar: $0.32 \pm 0.03,0.31 \pm 0.03,0.31 \pm 0.06$, and $0.33 \pm 0.07$, respectively. These values are similar to those found for nearby clusters which range from 0.30 to 0.40 (e.g., Devine et al. 2008). Therefore, no statistical difference could be found in the $K_{\mathrm{S}}$-band
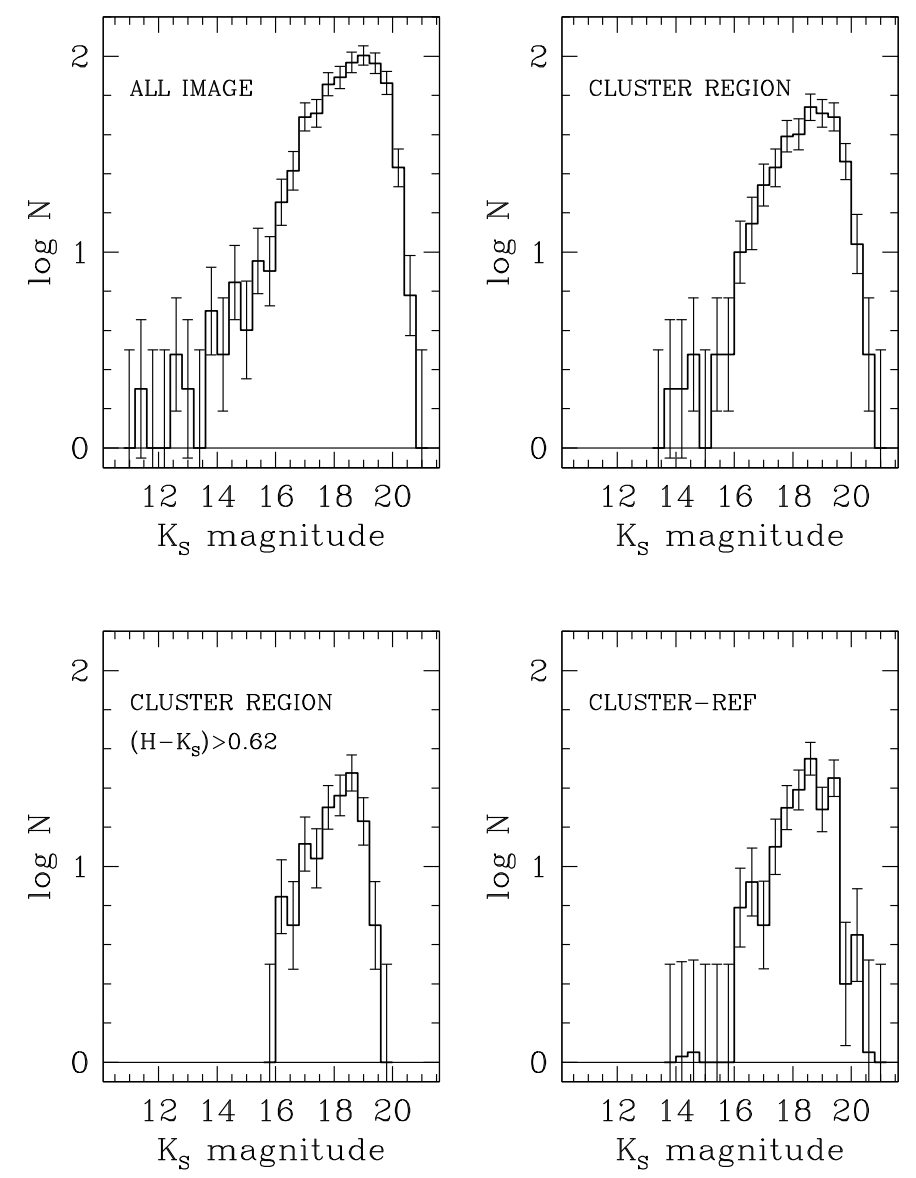

Fig. 13. Plots of the logarithm of the number of stars as a function of $K_{\mathrm{S}}$. The upper left panel is for all the stars in the $K_{\mathrm{S}}$-band image. The upper right panel is for the stars contained in the cluster region. The two lower panels are obtained from this panel: i) by restricting to the stars in the cluster region having $\left(H-K_{\mathrm{S}}\right)>0.62$ (lower left panel); ii) by subtracting the stars in a reference region (lower right panel). Error bars are $1 \sigma$ counting statistics.

luminosity function of this distant cluster located in the far outer Galaxy.

The slope found for this cluster seems to rule out a continuous star-formation scenario, favouring instead a coeval starformation case (Lada \& Lada 1995). In this case, the KLF has also been associated with cluster age, with KLFs of young clusters becoming shallower as they become older. The value of about 0.32 is compatible with an cluster age of about 4 Myr.

It is common to use the $K_{\mathrm{S}}$-band luminosity function to obtain the initial mass function IMF of a cluster. We have not attempted to do so because that conversion requires the knowledge of several factors involved, such as the pre-main-sequence massluminosity relationship, the population incompleteness, the extinction, the cluster age, and a proper background-source subtraction (Muench et al. 2000). None of these parameters is well known and would introduce large uncertainties into an IMF calculation.

Bearing in mind that we do not estimate any individual masses, but taking into consideration the above conclusions, we can make a rough estimate of the total mass present in the stellar content of this cluster. We assume that we have detected all stars down to $0.4 M_{\odot}$ and we use a Salpeter IMF down to $1 M_{\odot}$. Below $1 M_{\odot}$, we use a shallower slope (of -1.2 , Stahler \& Palla 2004). We obtain a total stellar mass of about $200 M_{\odot}$ inside the 
cluster region. Furthermore, assuming that clump A is the one that gave origin to the young stellar cluster, using the mass of this clump as the mass of the gas, we obtain a star-formation efficiency of $M_{\text {stars }} /\left(M_{\text {stars }}+M_{\mathrm{A}}\right) \simeq 5.7 \%$. Even though the uncertainty is large, these values are similar to other values of starformation efficiencies found in cluster environments within the local star-formation regions (e.g., L1630, Lada 1999).

The presence and properties of this molecular cloud containing this young stellar cluster at this location in the far outer Galactic disc constitutes further evidence that the properties of the far outer Galaxy molecular clouds and their star-formation activity are quite similar to those of molecular clouds in the inner Galaxy. This is also true for the few previous cases where we have found clusters in the far outer Galaxy: IRAS 07255-2012 (Santos et al. 2000), and IRAS 06361-0142 (Yun et al. 2007). Another common feature for these 3 clusters, all located in the far outer Galaxy, is the absence of very massive (O and B) stars. Albeit the small number statistics, there seems to be a tendency for distant far outer Galaxy molecular clouds not to form very massive stars.

\section{Summary}

We have discovered a new young stellar cluster seen towards a molecular cloud located at a distance of about $10 \mathrm{kpc}$ and a Galactocentric distance of about $15 \mathrm{kpc}$, well within the far outer Galaxy.

The cluster (with at least 136 objects) is detected in our nearinfrared images as a strong enhancement in the surface stellar density of our near-IR images. The cluster is contained in a region of about $4.0 \times 3.3 \mathrm{pc}^{2}$ centred close to IRAS $07527-3446$. It appears to consist of low and intermediate-mass young reddened stars with a large fraction having cleared the inner regions of their circumstellar discs. The observations are compatible with a $\leq 5 \mathrm{Myr}$ cluster with variable spatial extinction between $A_{\mathrm{V}}=5$ and $A_{\mathrm{V}}=11$. The slope of the $K_{\mathrm{S}}$-band luminosity function is estimated to be 0.33 , similar to those of young clusters in nearby star-forming regions and compatible with a coeval starformation scenario.

The molecular cloud, as traced by $\operatorname{CO}(J=1-0)$, extends over a region of $\sim 20 \times 15 \mathrm{pc}^{2}$. Our estimates of the mass of the molecular gas in this region, range from a few to several tens of thousands of solar masses, depending on the adopted technique and on the line transition used. In particular, decomposing the $\mathrm{CO}$ emission into clumps, we find that a clump is clearly associated with the cluster position, and has a mass of $3.3 \times 10^{3} M_{\odot}$. The average velocity dispersion of this clump suggests that it is not in virial equilibrium. In addition, the extinction $A_{\mathrm{V}}$ towards the cluster position, derived by means of different columndensity methods, was found to be in the range $A_{\mathrm{V}} \simeq 3-8$ mag. We estimate a value of $\sim 6 \%$ for the star-formation efficiency of this molecular region.

These findings confirm previous results that the distant outer Galaxy continues to be active in the production of new and rich stellar clusters, with the physical conditions required for the formation of rich clusters continuing to be met in the very distant environment of the outer Galactic disc.

Acknowledgements. This work has been partly supported by the Portuguese Fundação para a Ciência e Tecnologia (FCT) and by the European Commission FP6 Marie Curie Research Training Network "CONSTELLATION" (MRTNCT-2006-035890). This research made use of the NASA/ IPAC Infrared
Science Archive, which is operated by the Jet Propulsion Laboratory, California Institute of Technology, under contract with the National Aeronautics and Space Administration. This research also made use of the SIMBAD database, operated at CDS, Strasbourg, France, as well as SAOImage DS9, developed by the Smithsonian Astrophysical Observatory.

\section{References}

Adams, F. C., Lada, C. J., \& Shu, F. H. 1987, ApJ, 312, 788

Allen, C. W. 1973, Astrophysical Quantities, University of London (London: Athlone Press)

Bohlin, R. C., Savage, B. D., \& Drake, J. F. 1978, ApJ, 224, 132

Brand, J., \& Blitz, L. 1993, A\&A, 275, 67

Brand, J., \& Wouterloot, J. G. A. 1995, A\&A, 303, 851

Brand, J., \& Wouterloot, J. G. A. 2007, A\&A, 464, 909

Bessell, M. S., \& Brett, J. M. 1988, PASP, 100, 1143

Clemens, D. P., Sanders, D.B., \& Scoville, N.Z. 1988 , ApJ, 327, 139

Cutri, R. M., Skrutskie, M. F., Van Dyk, S., et al. 2003, Explanatory Supplement to the 2MASS All Sky Data Release, http://www.ipac.caltech.edu/ 2mass/releases/allsky/doc/explsup.html

Devine, K. E., Churchwell1, E. B., Indebetouw, R., Watson, C., \& Crawford, S. M. 2008, AJ, 135, 2095

Dickman, R. L. 1978, ApJS, 37, 407

Digel, S. W., Lyder, D. A., Philbrick, A. J., et al. 1996, ApJ, 458, 561

Elia, D., Massi, F., Strafella, F., et al. 2007, ApJ, 655, 316

Fich, M., \& Blitz, L. 1984, ApJ, 279, 125

Fich, M., Blitz, L., \& Stark, A.A. 1989, ApJ, 342, 272

Frerking, M. A., Langer, W. D., \& Wilson, R. W., 1982, ApJ, 262, 590

Haisch, K. E., Jr., Lada, E. A., \& Lada, C. J. 2001, ApJ, 553, L153

Heyer, M. H., Snell, R. L., Brunt, C., et al. 1998, ApJS, 115, 241

Horner, D. J., Lada, E. A., \& Lada, C. J. 1993, AJ, 113, 1788

Kobayashi, N., \& Tokunaga, A. T. 2000, ApJ, 532, 423

Koornneef, J. 1983, A\&A, 128, 84

Lada, E. A. 1999, The Origin of Star and Planetary Systems, ed. C. Lada, \& N. Kylafis (Dordrecht: Kluwer), 441

Lada, E. A., \& Lada, C. J. 1995, AJ, 109, 1682

Luhman, K. L., Rieke, G. H., Lada, C. J., \& Lada, E. A. 1998, ApJ, 508, 347

Luhman, K. L., Rieke, G. H., Young, E. T., et al. 2000, ApJ, 540, 1016

May, J., Alvarez, H., \& Bronfman, L. 1997, A\&A, 327, 325

McCaughrean, M. J., \& Stauffer, J. R. 1994, AJ, 108, 1382

Mead, K. N., \& Kutner, M. L. 1988, ApJ, 330, 399

Moorwood, A., Cuby, J.-G., Biereichel, P., et al. 1998, Messenger, 94, 7

Muench, A. A., Lada, E. A., \& Lada, C. J. 2000, ApJ, 533, 358

Muench, A. A., Lada, E. A., Lada, C. J., et al. 2003, AJ, 125, 2029

Padoan, P., Juvela, M., Bally, J., \& Nordlund, A. A., 2000, ApJ, 529, 259

Panagia, N. 1973, AJ, 78, 929

Pandey, A. K., Upadhyay, K., Ogura, K., et al. 2005, MNRAS, 358, 1290

Persson, S. E., Murphy, D. C., Krzeminski, W., Roth, M., \& Rieke, M. J. 1998, AJ, 116, 2475

Pineda, J. E., Caselli, P., \& Goodman, A. A. 2008, ApJ, 679, 481

Rieke, G. H., \& Lebofsky, M. J. 1985, ApJ, 288, 618

Rodríguez, L. F., Moran, J. M., Ho, P. T. P., \& Gottlieb, E. W. 1980, ApJ, 235, 845

Rudolph, A. L., Brand, J., de Geus, E. J., \& Wouterloot, J. G. A. 1996, ApJ, 458, 653

Santos, C. A., Yun, J. L., Clemens, D. P., \& Agostinho, R. J. 2000, ApJ, 540, 87

Siess L., Dufour E., \& Forestini M. 2000, A\&A, 358, 59

Skrutskie, M. F., Cutri, R. M., Stiening, R., et al. 2006, ApJ, 131, 1163

Snell, R. L., Carpenter, J. M., \& Heyer, M. H. 2002, ApJ, 578, 229

Stahler, S. W., \& Palla, F. 2004, The Formation of Stars (Wiley-VCH)

Strom, K. M., Strom, S. E., \& Merril, M. 1993, ApJ, 412, 233

Strong, A. W., \& Mattox, J. R. 1996, A\&A, 308, L21

Tapia, M., Lopéz, J. A., Rubio, M., Persi, P., \& Ferrari-Toniolo, M. 1991, A\&A, 242,388

Vesperini, E., \& Heggie, D. C. 1997, MNRAS, 289, 898

Williams, J. P., de Geus, E. J., \& Blitz, L. 1994, ApJ, 428, 693

Wilson, T. L., \& Matteucci, F. 1992, A\&AR, 4, 1

Wouterloot, J. G. A., \& Brand, J. 1989, A\&AS, 80, 149

Wouterloot, J. G. A., Brand, J., \& Henkel, C. 1988, A\&A, 191, 323

Wouterloot, J. G. A., Brand, J., Burton, W. B., \& Kwee, K. K. 1990, A\&A, 230, 21

Yasui, C., \& Kobayashi, N. 2008, ApJ, 675, 443.

Yun, J. L., Lopez-Sepulcre, A., \& Torrelles, J. M. 2007, A\&A, 471, 573 
J. L. Yun et al.: Gas and new young stars in the far outer Galaxy, Online Material $p 1$

Table 2. Photometry of cluster members.

\begin{tabular}{|c|c|c|c|c|c|}
\hline ID & $\begin{array}{c}\text { RA } \\
(2000)\end{array}$ & $\begin{array}{c}\text { Dec } \\
(2000)\end{array}$ & $m_{K_{\mathrm{S}}}$ & $\overline{\left(H-K_{\mathrm{S}}\right)}$ & $\overline{\left(J-K_{\mathrm{S}}\right)}$ \\
\hline 1 & 075434.22 & -34553.2 & 18.6 & 0.62 & 1.61 \\
\hline 2 & 075435.60 & -34551.2 & 18.2 & 0.81 & 2.53 \\
\hline 3 & 075435.39 & -34551.3 & 18.4 & 0.73 & 2.26 \\
\hline 4 & 075435.67 & -345459.8 & 19.5 & 0.73 & 9.99 \\
\hline 5 & 075436.08 & -345457.6 & 18.8 & 0.65 & 2.17 \\
\hline 6 & 075435.49 & -345457.4 & 19.3 & 0.65 & 2.07 \\
\hline 7 & 075435.28 & -345456.2 & 18.5 & 0.73 & 1.99 \\
\hline 8 & 075434.84 & -345456.3 & 18.2 & 0.76 & 2.12 \\
\hline 9 & 075435.98 & -345455.7 & 18.0 & 0.70 & 2.09 \\
\hline 10 & 075435.90 & -345455.4 & 18.4 & 0.70 & 9.99 \\
\hline 11 & 075435.55 & -345454.9 & 19.3 & 0.80 & 9.99 \\
\hline 12 & 075436.10 & -345454.2 & 18.8 & 0.92 & 2.08 \\
\hline 13 & 075435.50 & -345454.2 & 17.7 & 1.30 & 3.16 \\
\hline 14 & 075435.68 & -345453.6 & 17.4 & 1.01 & 2.69 \\
\hline 15 & 075435.60 & -345453.5 & 18.6 & 0.86 & 2.51 \\
\hline 16 & 075435.91 & -345453.4 & 16.5 & 0.84 & 2.19 \\
\hline 17 & 075435.72 & -345453.1 & 18.5 & 0.93 & 9.99 \\
\hline 18 & 075436.48 & -345452.6 & 18.2 & 0.74 & 2.25 \\
\hline 19 & 075436.16 & -345452.6 & 17.2 & 1.57 & 9.99 \\
\hline 20 & 075435.82 & -345452.6 & 17.6 & 0.80 & 2.30 \\
\hline 21 & 075435.73 & -345452.2 & 17.1 & 1.68 & 3.78 \\
\hline 22 & 075436.39 & -345452.2 & 19.1 & 1.12 & 9.99 \\
\hline 23 & 075436.30 & -345452.1 & 16.9 & 1.18 & 3.05 \\
\hline 24 & 075436.07 & -345451.5 & 17.9 & 0.62 & 1.86 \\
\hline 25 & 075435.41 & -345451.7 & 16.5 & 1.02 & 2.34 \\
\hline 26 & 075435.81 & -345451.3 & 16.7 & 0.93 & 2.70 \\
\hline 27 & 075435.52 & -345451.4 & 18.5 & 1.04 & 2.35 \\
\hline 28 & 075432.78 & -345451.2 & 18.5 & 0.95 & 2.40 \\
\hline 29 & 075436.13 & -345451.1 & 16.9 & 0.86 & 2.38 \\
\hline 30 & 075437.76 & -345450.7 & 17.6 & 0.93 & 2.41 \\
\hline 31 & 075435.45 & -345450.9 & 18.2 & 1.00 & 2.34 \\
\hline 32 & 075435.97 & -345450.4 & 17.8 & 0.83 & 2.32 \\
\hline 33 & 075435.66 & -345450.4 & 17.9 & 1.09 & 9.99 \\
\hline 34 & 075435.79 & -345450.3 & 17.8 & 0.91 & 2.24 \\
\hline 35 & 075435.62 & -345449.9 & 18.3 & 0.83 & 1.94 \\
\hline 36 & 075436.00 & -345449.8 & 16.5 & 1.09 & 2.79 \\
\hline 37 & 075435.78 & -345449.7 & 17.1 & 0.78 & 9.99 \\
\hline 38 & 075436.25 & -345449.6 & 18.4 & 0.69 & 2.06 \\
\hline 39 & 075435.88 & -345449.5 & 18.1 & 0.66 & 9.99 \\
\hline 40 & 075435.50 & -345449.5 & 17.2 & 0.73 & 2.23 \\
\hline 41 & 075436.72 & -345448.9 & 18.4 & 0.66 & 1.89 \\
\hline 42 & 075434.50 & -345448.7 & 18.0 & 0.64 & 2.07 \\
\hline 43 & 075435.66 & -345448.4 & 16.9 & 0.96 & 9.99 \\
\hline 44 & 075436.07 & -345448.3 & 16.6 & 0.66 & 2.03 \\
\hline 45 & 075435.99 & -345448.2 & 17.9 & 0.92 & 9.99 \\
\hline 46 & 075435.77 & -345448.2 & 12.5 & 1.04 & 2.16 \\
\hline 47 & 075436.90 & -345448.2 & 17.8 & 1.08 & 2.84 \\
\hline 48 & 075436.52 & -345448.1 & 19.0 & 0.65 & 1.63 \\
\hline 49 & 075435.63 & -345447.9 & 16.2 & 1.12 & 2.57 \\
\hline 50 & 075435.21 & -345447.8 & 18.7 & 1.13 & 9.99 \\
\hline 51 & 075436.16 & -345447.7 & 18.3 & 0.79 & 1.91 \\
\hline 52 & 075437.63 & -345447.6 & 18.5 & 0.75 & 2.04 \\
\hline 53 & 075436.23 & -345447.5 & 18.3 & 0.90 & 2.25 \\
\hline 54 & 075435.53 & -345447.6 & 18.9 & 0.88 & 9.99 \\
\hline 55 & 075435.34 & -345447.5 & 19.0 & 0.92 & 2.85 \\
\hline 56 & 075436.01 & -345447.0 & 18.2 & 1.10 & 9.99 \\
\hline 57 & 075436.87 & -345446.9 & 18.6 & 0.97 & 9.99 \\
\hline 58 & 075434.67 & -345446.9 & 17.1 & 0.65 & 2.12 \\
\hline 59 & 075435.21 & -345446.7 & 18.7 & 0.64 & 2.01 \\
\hline
\end{tabular}


J. L. Yun et al.: Gas and new young stars in the far outer Galaxy, Online Material $p 2$

Table 2. continued.

\begin{tabular}{|c|c|c|c|c|c|}
\hline ID & $\begin{array}{c}\text { RA } \\
(2000)\end{array}$ & $\begin{array}{c}\text { Dec } \\
(2000)\end{array}$ & $m_{K_{\mathrm{S}}}$ & $\left(H-K_{\mathrm{S}}\right)$ & $\left(J-K_{\mathrm{S}}\right)$ \\
\hline 60 & 075436.07 & -345446.5 & 18.7 & 0.89 & 2.39 \\
\hline 61 & 075434.42 & -345446.3 & 17.5 & 0.76 & 2.49 \\
\hline 62 & 075436.79 & -345446.1 & 18.2 & 1.06 & 2.78 \\
\hline 63 & 075435.08 & -345446.3 & 18.6 & 0.85 & 2.70 \\
\hline 64 & 075436.92 & -345446.0 & 16.2 & 0.69 & 1.86 \\
\hline 65 & 075437.12 & -345445.9 & 18.7 & 0.68 & 2.10 \\
\hline 66 & 075435.79 & -345445.7 & 17.8 & 0.64 & 1.74 \\
\hline 67 & 075436.67 & -345445.6 & 17.6 & 1.23 & 9.99 \\
\hline 68 & 075436.05 & -345445.7 & 18.2 & 0.71 & 2.12 \\
\hline 69 & 075436.59 & -345445.3 & 15.4 & 1.34 & 2.80 \\
\hline 70 & 075434.61 & -345445.1 & 16.1 & 1.35 & 3.06 \\
\hline 71 & 075437.07 & -345444.8 & 18.1 & 0.73 & 2.04 \\
\hline 72 & 075436.88 & -345445.0 & 18.1 & 0.88 & 1.97 \\
\hline 73 & 075436.21 & -345444.5 & 18.2 & 0.64 & 1.91 \\
\hline 74 & 075434.41 & -345444.3 & 17.2 & 1.09 & 2.84 \\
\hline 75 & 075435.50 & -345444.0 & 18.6 & 0.86 & 2.11 \\
\hline 76 & 075436.73 & -345443.5 & 18.2 & 1.03 & 2.58 \\
\hline 77 & 075436.04 & -345443.2 & 16.2 & 1.19 & 2.77 \\
\hline 78 & 075436.80 & -345443.1 & 18.2 & 0.98 & 2.56 \\
\hline 79 & 075436.57 & -345442.8 & 18.5 & 1.06 & 2.65 \\
\hline 80 & 075436.70 & -345442.5 & 17.9 & 1.06 & 2.74 \\
\hline 81 & 075437.17 & -345442.1 & 18.0 & 1.04 & 2.43 \\
\hline 82 & 075436.54 & -345442.1 & 18.4 & 0.91 & 2.38 \\
\hline 83 & 075436.21 & -345442.0 & 17.9 & 0.79 & 2.48 \\
\hline 84 & 075436.11 & -345441.9 & 18.9 & 0.84 & 2.13 \\
\hline 85 & 075436.99 & -345441.8 & 13.9 & 0.79 & 1.80 \\
\hline 86 & 075438.05 & -345441.5 & 18.7 & 0.74 & 1.94 \\
\hline 87 & 075437.49 & -345441.4 & 18.5 & 0.68 & 2.09 \\
\hline 88 & 075437.11 & -345441.4 & 17.3 & 0.65 & 1.91 \\
\hline 89 & 075437.87 & -345441.4 & 18.7 & 0.70 & 2.07 \\
\hline 90 & 075436.27 & -345440.9 & 16.9 & 0.94 & 2.66 \\
\hline 91 & 075435.72 & -345441.0 & 19.5 & 0.72 & 9.99 \\
\hline 92 & 075435.02 & -345440.9 & 19.2 & 0.89 & 9.99 \\
\hline 93 & 075434.94 & -345440.4 & 18.4 & 0.83 & 2.38 \\
\hline 94 & 075436.58 & -345440.2 & 18.2 & 1.05 & 2.58 \\
\hline 95 & 075435.51 & -345440.1 & 19.1 & 0.82 & 9.99 \\
\hline 96 & 075435.63 & -345440.0 & 18.5 & 0.95 & 2.58 \\
\hline 97 & 075437.66 & -345439.8 & 17.5 & 0.73 & 2.18 \\
\hline 98 & 075436.51 & -345439.9 & 17.3 & 1.13 & 2.96 \\
\hline 99 & 075436.06 & -345439.6 & 17.8 & 0.87 & 2.41 \\
\hline 100 & 075437.27 & -345439.4 & 17.1 & 0.68 & 2.10 \\
\hline 101 & 075437.08 & -345439.3 & 19.0 & 0.76 & 1.94 \\
\hline 102 & 075434.39 & -345439.2 & 18.5 & 0.65 & 2.19 \\
\hline 103 & 075437.42 & -345439.0 & 18.4 & 0.85 & 2.17 \\
\hline 104 & 075437.51 & -345438.8 & 18.9 & 0.67 & 1.91 \\
\hline 105 & 075436.15 & -345438.8 & 17.3 & 0.65 & 1.88 \\
\hline 106 & 075435.97 & -345438.8 & 18.4 & 0.75 & 1.96 \\
\hline 107 & 075437.09 & -345438.4 & 17.3 & 0.73 & 2.26 \\
\hline 108 & 075436.31 & -345438.0 & 18.9 & 0.74 & 1.82 \\
\hline 109 & 075436.21 & -345438.0 & 16.2 & 0.92 & 2.13 \\
\hline 110 & 075437.56 & -345437.9 & 19.0 & 0.75 & 2.34 \\
\hline 111 & 075436.20 & -345437.6 & 15.9 & 0.92 & 2.29 \\
\hline 112 & 075435.57 & -345437.6 & 19.3 & 0.79 & 9.99 \\
\hline 113 & 075434.30 & -345437.6 & 17.1 & 1.14 & 2.96 \\
\hline 114 & 075437.35 & -345436.7 & 17.8 & 1.07 & 2.40 \\
\hline 115 & 075437.88 & -345436.2 & 16.3 & 0.85 & 2.26 \\
\hline 116 & 075437.39 & -345435.9 & 14.3 & 1.33 & 3.16 \\
\hline 117 & 075435.99 & -345434.9 & 17.6 & 0.77 & 2.23 \\
\hline 118 & 075436.94 & -345434.5 & 17.1 & 0.77 & 2.17 \\
\hline 119 & 075436.27 & -345434.4 & 18.7 & 0.68 & 2.09 \\
\hline
\end{tabular}


J. L. Yun et al.: Gas and new young stars in the far outer Galaxy, Online Material $p 3$

Table 2. continued.

\begin{tabular}{cccccc}
\hline \hline ID & $\begin{array}{c}\text { RA } \\
(2000)\end{array}$ & $\begin{array}{c}\text { Dec } \\
(2000)\end{array}$ & $m_{K_{\mathrm{S}}}$ & $\left(H-K_{\mathrm{S}}\right)$ & $\left(J-K_{\mathrm{S}}\right)$ \\
\hline 120 & 075437.85 & -345433.6 & 18.6 & 0.63 & 1.83 \\
121 & 075436.35 & -345432.4 & 19.2 & 0.91 & 2.25 \\
122 & 075437.59 & -345432.3 & 18.1 & 0.75 & 2.17 \\
123 & 075435.51 & -345431.9 & 17.6 & 0.69 & 2.16 \\
124 & 075435.88 & -345430.8 & 17.4 & 0.82 & 2.15 \\
125 & 075434.76 & -345430.5 & 18.7 & 0.67 & 2.07 \\
126 & 075433.11 & -345429.9 & 18.8 & 0.64 & 1.89 \\
127 & 075436.26 & -345429.7 & 17.9 & 1.24 & 2.90 \\
128 & 075437.27 & -345429.5 & 17.1 & 0.75 & 2.09 \\
129 & 075436.67 & -345428.5 & 18.5 & 0.66 & 1.98 \\
130 & 075433.11 & -345425.3 & 19.1 & 0.70 & 1.69 \\
131 & 075439.24 & -345424.9 & 18.4 & 0.84 & 2.48 \\
132 & 075437.18 & -345424.3 & 18.2 & 1.02 & 2.78 \\
133 & 075437.90 & -345418.6 & 19.0 & 0.63 & 2.19 \\
134 & 075437.37 & -345418.1 & 16.4 & 0.75 & 1.96 \\
135 & 075437.01 & -345416.0 & 17.3 & 0.66 & 1.92 \\
136 & 075437.16 & -345412.8 & 18.0 & 0.84 & 2.49 \\
\hline
\end{tabular}

